\title{
3-Hydroxypyrimidine-2,4-dione-5- $\mathrm{N}$-benzylcarboxamides potently inhibit HIV-1 integrase and RNase H
}

\author{
Bulan Wu ${ }^{a}$, Jing Tang ${ }^{a}$, Daniel J. Wilson ${ }^{a}$, Andrew D. Huber ${ }^{b}$, Mary C. Casey ${ }^{b}$, Juan $\mathbf{J i}^{\mathrm{b}}$, \\ Jayakanth Kankanala ${ }^{a}$, Jiashu Xie ${ }^{a}$, Stefan G. Sarafianos ${ }^{b}$, and Zhengqiang Wang ${ }^{\star}$,a \\ ${ }^{a}$ Center for Drug Design, Academic Health Center, University of Minnesota, Minneapolis, MN \\ 55455, USA \\ bepartment of Molecular Microbiology and Immunology and Department of Biochemistry, \\ University of Missouri School of Medicine, Christopher S. Bond Life Sciences Center, Columbia, \\ MO 65211, USA
}

\begin{abstract}
Resistance selection by human immunodeficiency virus (HIV) towards known drug regimens necessitates the discovery of structurally novel antivirals with a distinct resistance profile. Based on our previously reported 3-hydroxypyrimidine-2,4-dione (HPD) core we have designed and synthesized a new integrase strand transfer (INST) inhibitor type featuring a 5- $N$ benzylcarboxamide moiety. Significantly, the 6-alkylamino variant of this new chemotype consistently conferred low nanomolar inhibitory activity against HIV-1. Extended antiviral testing against a few raltegravir-resistant HIV-1 clones revealed a resistance profile similar to that of the second generation INST inhibitor (INSTIs) dolutegravir. Although biochemical testing and molecular modeling also strongly corroborate the inhibition of INST as the antiviral mechanism of action, selected antiviral analogues also potently inhibited reverse transcriptase (RT) associated RNase H, implying potential dual target inhibition. In vitro ADME assays demonstrated that this novel chemotype possesses largely favorable physicochemical properties suitable for further development.
\end{abstract}

\section{Introduction}

HIV infects an estimated 34 million people worldwide. Clinical management of HIV / AIDS continues to rely on highly active antiretroviral therapy (HAART) that consists of five different classes of FDA-approved drugs ${ }^{1}$ : nucleoside reverse transcriptase inhibitors (NRTIs); nonnucleoside reverse transcriptase inhibitors (NNRTIs); protease inhibitors (PIs); INSTIs; and entry inhibitors. However, HIV infection remains incurable ${ }^{2-3}$ due to the persistence of viral reservoirs. The resulting long duration of HAART typically amounts to two treatment challenges: toxicities and the selection of resistant viral strains. The emergence of multidrug resistance is particularly concerning as treatment options will be

\footnotetext{
*Corresponding Author wangx472@umn.edu; Phone: +1 (612) 626-7025.

Supporting Information Available. Synthesis and characterization data, including ${ }^{1} \mathrm{H}$ NMR, ${ }^{13} \mathrm{C}$ NMR and HRMS data, of intermediates 7-23. This material is available free of charge via the Internet at http://pubs.acs.org.
} 
severely limited. Therefore, sustained success in HIV HAART requires novel antivirals with structurally unique pharmacophore and distinct resistance profile. HIV IN plays critical roles in viral infection and the establishment of proviral latency. ${ }^{4-6}$ As an antiviral target IN is particular attractive because 1) there is no host cellular counterpart, hence specific inhibitors should not interfere with cellular functions; and 2) IN uses the same active site (DD35E motif) for both the 3' processing and the ST steps, therefore, inhibitors could benefit from a potentially high genetic barrier to resistance selection. Specific INSTIs all feature a diketoacid (DKA) functionality or its heterocyclic bioisosteres ${ }^{7-11}$ along with a hydrophobic terminal benzyl moiety, ${ }^{12-17}$ as demonstrated by all three FDA-approved INSTIs (Figure 1): raltegravir (1) ${ }^{18-19}$, elvitegravir (2), ${ }^{20}$ and dolutegravir (3). ${ }^{21-22}$ Particularly significant is the second-generation INSTI 3 which retains potency against many raltegravir-resistant HIV strains. ${ }^{23}$ We have previously developed a few chemotypes featuring the HPD core ${ }^{24-27}$ that effectively inhibited HIV-1 in cell culture. The antiviral potency associated with these HPD subtypes is likely due to the dual inhibition of RT and IN as indicated by biochemical assays. However, the IN inhibition was typically much weaker than the inhibition of RT. Furthermore, cross resistance to $\mathbf{1}$ was also observed, suggesting that these early HPD subtypes may have the characteristics of first-generation INSTIs. Another variant of HPD was recently found to selectively inhibit the RT-associated RNase $\mathrm{H}$ without significantly inhibiting INST. ${ }^{28}$ We report herein a rationally designed new HPD variant (Figure 1, 4) featuring a unique C5 carboxamide moiety to specifically inhibit INST. Significantly, chemotype $\mathbf{4}$ has the two structural determinants essential for INST binding and inhibition (Figure 1). The overall shape and functionalities of $\mathbf{4}$ particularly resemble those of $\mathbf{3}$, suggesting that our novel inhibitors can be second generation INSTIs.

\section{Results and Discussion}

\section{Chemistry}

Our target compounds 4 were prepared via a concise and diverse synthetic route shown in Scheme 1. The synthesis started from commercially available hydroxyurea 5 which was $O$ benzylated with $\mathrm{BnBr}$ in the presence of $\mathrm{KOH}$ under reflux to provide 1-(benzyloxy)urea 6 in high yield. ${ }^{29}$ Cyclocondensation of 1-(benzyloxy)urea 2 and diethyl malonate provided six-membered heterocycle compound 3-(benzyloxy)-6-hydroxypyrimidine-2,4(1H,3H)dione 7 in moderate yield under microwave irradiation $\left(150{ }^{\circ} \mathrm{C}, 20 \mathrm{~min}\right)$ or under conventional heating (reflux, overnight). ${ }^{30}$ Compound 7 was converted to key intermediate 3-(benzyloxy)-6-chloropyrimidine-2,4(1H,3H)-dione 8 by reacting with $\mathrm{POCl}_{3}$ in the presence of $\mathrm{BnEt}_{3} \mathrm{NCl}^{31}$ Reaction of intermediate chloride $\mathbf{8}$ with amines in the presence of $\mathrm{N}, \mathrm{N}$-dimethylaniline under microwave condition provided 6-amination products 9 in moderate to good yield. ${ }^{32}$ The key $\mathrm{C} 5$ carboxamide was introduced by reacting 6-amino intermediates 9 with commercial or in situ generated isocyanates (Scheme 1), ${ }^{33}$ a highly efficient method for small scale synthesis of intermediate 10. The final debenzylation was achieved by treating compounds 10 with TFA under microwave condition ${ }^{34}$ or via catalytic hydrogenation.

Alternatively, the $\mathrm{C} 5$ carboxamidation can be achieved via a two-step reaction sequence (scheme 2) to avoid the use of the unpleasant nitrobenzene. In this case, the amino 
intermediate 9 was first treated with phenyl chloroformate and a base, such as pyridine, to give intermediate phenyl ester $\mathbf{1 1}$ which was converted to amide $\mathbf{1 0}$ upon reacting with a primary amine under conventional heating or microwave conditions. Debenzylation with the same protocol afforded the desired chemotype 4 .

Meanwhile our analogue synthesis also included a few variants of $\mathbf{4}$ which entailed slightly different synthetic routes or further functionalization (Scheme 3). In these events, direct debenzylation of intermediate $\mathbf{1 2}$ afforded compound $\mathbf{5 1}$ (Scheme 3, a), whereas methylation of intermediate 13 produced two regio-isomers 14 and $\mathbf{1 5}$, which upon debenzylation yielded compounds $\mathbf{5 2}$ and $\mathbf{5 3}$, respectively (Scheme 3, b). Interestingly, a 6-deamino analogue 54 was also synthesized from intermediate $\mathbf{7}$ via the carboxamidation and debenzylation sequence (Scheme 3, c).

Finally, synthesis of analogues (55-57) with another six-membered ring fused to the HPD core via N1-C6 (55) or C6-C5 (56-57) was also attempted. Compound 55 was synthesized from 6-amino HPD intermediate $\mathbf{1 7}$ which was cyclized to $\mathbf{1 8}$ upon treating with 1,3dibromopropane under basic condition (Scheme 4). The rest of the synthesis involved the same carboxamidation-debenzylation sequence as used for the synthesis of chemotype 4 . The synthesis of C6-C5 fused analogues (56-57) was attempted based on Scheme 5. While the two intermediates 22-23 were obtained, the subsequent debenzylation was unsuccessful (Scheme 5).

\section{Biology}

All final compounds (24-55) were first screened in a viral cytopathic effect (CPE) based antiviral assay against HIV-1IIIB in CEM-SS cells. ${ }^{35-36}$ In this assay, the reduction of CPE was used to indicate the antiviral activity of a compound. The screening was conducted under single concentration $(10 \mu \mathrm{M})$ and the percent inhibition and percent cell viability were calculated using a virus control and cell control, respectively. Best compounds from this assay were further evaluated in a single replication cycle MAGI assay. In addition, all compounds were tested in a biochemical assay with recombinant HIV-1 IN measuring INST activity. Selected ones were also tested against RT-associated RNase $\mathrm{H}$ activity. Both $\mathbf{3}$ and $\mathbf{1}$ were included in all assays as reference compounds. The results indicate a few prominent structure-activity-relationship (SAR) trends.

Alkylamino group is required at C6-From the antiviral screening assay, the most striking SAR was that the two analogues with an aromatic amine at the C6 position (24-25) were completely inactive and largely cytotoxic in the cytoprotection antiviral assay, whereas a small aliphatic amine substitution at the same position (26-37, Table 1) conferred excellent antiviral activity without cytotoxicity, with the lone exception being the butylamino analogue (42) which was inactive and cytotoxic. The same dramatic SAR was observed from the INST biochemical assay in which neither of the arylamino analogues (24-25) showed appreciable inhibition at concentrations up to $100 \mu \mathrm{M}$ whereas the alkylamino analogues (26-37) all demonstrated nanomolar inhibitory activity, including the tbutylamino analogue (35). Furthermore, although most alkyl analogues (26-31, 33-34) produced maximal cytoprotection at $10 \mu \mathrm{M}$ without significant cytotoxicity, linear alkyl substituted analogues 
(26-31) are generally favored in the INST biochemical assay over the unsubstituted analogue (36) and the bulky alkyl substituted ones, such as the isopropyl (33) and cyclopropyl (34) groups. Biochemical analysis also showed that the ethyl substituent (30) confers the best inhibition against INST $\left(\mathrm{IC}_{50}=33 \mathrm{nM}\right)$.

$\mathrm{N}$-Benzyl substitution on the $\mathbf{C} 5$ carboxamide is highly desired-To explore the SAR concerning the C5 carboxamide moiety, analogues of compound $\mathbf{3 0}$ were synthesized and tested in the antiviral screening assay and the INST biochemical assay (Table 2). Remarkably, all analogues with a benzyl group on the C5 carboxamide $\mathbf{3 0}, \mathbf{3 7}$ 46) demonstrated maximal cytoprotection (92-100\%) without considerable cytotoxicity (cell viability $87-100 \%$ ), with the only exception being compound 43 which provided $62 \%$ cytoprotection with $73 \%$ cell viability at $10 \mu \mathrm{M}$. Consistent with the maximal cytoprotection, exceptional biochemical inhibition $\left(\mathrm{IC}_{50}=21-230 \mathrm{nM}\right)$ was also observed for these analogues. Interestingly, compound with an extra methylene group (47) also produced maximal cytoprotection at $10 \mu \mathrm{M}$, though the biochemical inhibition was substantially reduced (47 vs $\mathbf{3 0}$ ). In stark contrast, replacing the benzyl group with a phenyl ring (48), alkyl group (50), or removing the $N$-substituent (49) completely abrogated both the antiviral activity and biochemical inhibition. Compound with a C5 phenyl ester (51) was also devoid of biochemical inhibition and yielded $50 \%$ cytoprotection at $10 \mu \mathrm{M}$. All these SAR observations are well aligned with the known pharmacophore model of INSTIs.

Scaffold analogues lack antiviral activity or biochemical INST inhibition-To further confirm the SAR trends shown in Tables 1-2, we also synthesized a few scaffold analogues of the new chemotype. Compounds 52-53 were synthesized to explore the impact of methylation on $\mathrm{N} 1$ or the C5 carboxamide. Remarkably, a simple methylation at N1 completely abrogated the antiviral activity, cell viability and the biochemical inhibition (52). Methylation of the C5 carboxamide also caused a complete loss of antiviral activity while substantially attenuating the biochemical inhibition (53 vs 38). Similarly, the 6-deamino analogue 61 demonstrated drastically reduced antiviral potency and biochemical inhibition (54 vs 30), confirming the importance of the amino group at C6 for biological activities. Finally, cyclized analogue $\mathbf{5 5}$ was inactive and cytotoxic in the antiviral screening assay, though the biochemical inhibition was largely preserved $\left(\mathrm{IC}_{50}=430 \mathrm{nM}\right)$.

Dose response antiviral testing-To better gauge the antiviral potency, selected inhibitors with excellent $\mathrm{CPE}$ reduction and cell viability from the antiviral screening assay were further tested in a dose response fashion, from which the $\mathrm{EC}_{50}$ and $\mathrm{CC}_{50}$ were determined (Table 4). All compounds tested showed nanomolar activity and five of the eight analogues were active in low nanomolar range $\left(\mathrm{EC}_{50}=15-61 \mathrm{nM}\right)$. Although these analogues showed mild cytotoxicity with $\mathrm{CC}_{50}$ s in the range of $18-58 \mu \mathrm{M}$, the selectivity window (Table 4), particularly that for analogues 40 (TI =1500) and 45 (TI = 1200), is sufficiently large to warrant their further development. To confirm the observed antiviral potency, these same analogues were further evaluated in a distinct MAGI assay. ${ }^{36-37}$ This assay measures HIV infection in indicator cells (P4R5) through the expression of a Tatdependent reporter ( $\beta$-galactosidase) and requires only one replication cycle. While the $\mathrm{EC}_{50}$ values were generally higher in this assay, our compounds still demonstrated nanomolar 
antiviral potency with the exception of compounds 26 and $\mathbf{4 6}$. Interestingly, compound $\mathbf{4 5}$, while showing the best potency in both the INST assay and the CPE antiviral assay, exhibited a significantly higher $\mathrm{EC}_{50}(0.30 \mu \mathrm{M}$ vs $0.015 \mu \mathrm{M})$ in the MAGI assay. By comparison, $\mathrm{EC}_{50}$ values for analogues $\mathbf{3 8}$ and $\mathbf{4 0}$ were comparable to $\mathbf{1}$ in the CPE assay and only 2-5 fold higher than $\mathbf{3}$ and $\mathbf{1}$ in the MAGI assay. Nevertheless, dose response antiviral testing in two distinct assays confirmed a highly potent antiviral profile of our newly designed compounds.

Antiviral resistance profile-Mutations conferring resistance to INSTIs have been generated in cell culture and emerged in clinical studies. ${ }^{38-40}$ To establish the resistance profile of chemotype $\mathbf{4}$, a representative compound $\mathbf{4 5}$ was evaluated for antiviral potency against four raltegravir-resistant HIV- 1 clones $^{41}$ in the CPE assay. These clones contain one or more major mutations in HIV-1 IN that are associated with raltegravir resistance, ${ }^{23,} 24$ namely a Y143C or an N155H single mutation; a G140S / Q148H double mutation and a G140S / Y143H / Q148H triple mutation. AZT, 1 and $\mathbf{3}$ were included in the study for comparison purpose. The results are summarized in Table 5. As expected cross-resistance to 1 was not observed for AZT due to its orthogonal mechanism of action as an NRTI, whereas the resistance profile observed in our antiviral assay for $\mathbf{3}$ is largely consistent with the reported profile. ${ }^{23}$ Similar to 3 , compound $\mathbf{4 5}$ retains its potency against clones with only one major IN mutation (clones 1556-1 and 4736-2) while HIV-1 clones with IN double mutations (clone 8070-1) and triple mutations (clone 8070-1) demonstrated moderate resistance to 45, with fold resistance of 13 and 6.3, respectively. The fold-resistance of $\mathbf{4 5}$ is strikingly similar to that observed with $\mathbf{3}$ for each of the four HIV-1 clones (Table 5). These observations strongly suggest that our chemotype $\mathbf{4}$ may confer antiviral activity via inhibiting INST in a manner similar to that of $\mathbf{3}$, a second generation INSTI. ${ }^{42}$

Inhibition against RT-associated RNase $\mathbf{H}$-Consistent with the aforementioned antiviral profile against raltegravir-resistant HIV-1 clones, which implies an antiviral mechanism of action (MOA) similar to second generation INSTI 3, biochemical data also closely correlate with the observed antiviral activity, as each of the selected analogues exhibited a biochemical $\mathrm{IC}_{50}$ against INST very similar to the antiviral $\mathrm{EC}_{50}$, particularly from the CPE assay (Table 6). In addition, none of the inactive compounds (24-25, 48 and 50-54) from the CPE antiviral assay inhibited INST (Tables 1-3), strongly suggesting that our new compounds target INST. However, HIV IN and RNase $\mathrm{H}$ share a similar active site fold ${ }^{43}$ hence their inhibition entails similar pharmacophore elements, mainly a chelating triad and a hydrophobic aromatic moiety. Therefore, we also tested selected analogues in another biochemical assay measuring the RNase $\mathrm{H}$ function of RT. Remarkably, all these analogues demonstrated exceptional biochemical inhibition against $\mathrm{RNase}_{\mathrm{H}}$ with $\mathrm{IC}_{50} \mathrm{~s}$ in the low nanomolar range (10-61 nM, Table 6), which correlates with the antiviral activity as well as the INST inhibition does in general. By contrast, the two reference compounds, 3 and 1, did not inhibit RNase $\mathrm{H}$ significantly at concentrations up to $10 \mu \mathrm{M}$. These observations indicate that the exceptional antiviral potency of our new chemotype could be due to dual target inhibition. Further virological characterizations of our compounds, including MOA studies via viral passage experiments for resistance selection, are currently underway and will be reported in due course. 


\section{Mechanism of strand transfer inhibition}

HIV IN is a $32-\mathrm{kDa}$ protein encoded by viral pol gene consisting of three functional domains ${ }^{44}$ : the N-terminal domain (NTD) with a conserved "HH-CC" zinc-binding motif; the catalytic core domain (CCD) containing the key D64-D116-E152 catalytic triad; and the C-terminal domain (CTD) important for DNA binding. Although crystal structures of each single domain as well as double domains had been reported ${ }^{45-47}$ detailed understanding on HIV INST catalysis and inhibition remained elusive until the disclosure of crystal structures of full-length IN and viral DNA complex (intasome) for a homologous prototype foamy virus (PFV). ${ }^{48-49}$ Homologous models ${ }^{50-51}$ constructed based on these PFV intasome crystal structures have provided valuable details into HIV INST mechanism of action and formed the basis for structure-based INSTI design. Significantly these models corroborate the minimal pharmacophore embedded in major INSTIs as shown in Figure 1.

To illustrate the binding mode of our new chemotype at HIV IN active site, we have performed molecular docking on a representative analogue $\mathbf{4 5}$ using a PFV intasome model (Figure 2). ${ }^{50}$ Significantly, $\mathbf{4 5}$ fits perfectly into the IN binding site through two major binding domains: the 3-N hydroxyl group simultaneously chelates to both $\mathrm{Mg}^{2+}$ ions while allowing the placement of the benzyl group into the protein-DNA interfacial hydrophobic pocket involving $\pi$-stacking of the i) fluorobenzyl side chain with the deoxycytosine $\mathrm{C} 16$ of viral DNA, and ii) $\pi$-stacking of the central uracil ring with the terminal $3^{\prime}$-deoxyadenosine A17 (Figure 2a). In addition, a structure overlay of $\mathbf{4 5}$ with $\mathbf{3}$ reveals a high degree of similarity between their binding (Figure 2b), suggesting that our new molecular scaffold can effectively engage with HIV IN for ST inhibition.

To understand the effect of mutations on antiviral profile of $\mathbf{1}$ and $\mathbf{4 5}$, molecular modeling was performed with these two compounds into the PFV IN Y212C mutant which corresponds to the Y143C of HIV IN (Figure 3). It was observed that the oxadiazole moiety of 1 makes a critical $\pi-\pi$ stack interaction with Y143 (Figure 3a). This interaction is not available with the $\mathrm{C} 143$ residue and the binding of $\mathbf{1}$ to the $\mathrm{Y} 143 \mathrm{C}$ mutant is significantly weakened (Figure 3b). On the other hand, 45 lacks the oxadiazole moiety to interact with Y143 (Figure 3a). Consequently, the Y143C mutation would not impact its binding to IN. These docking studies are consistent with the observations that Y143 mutation confers major resistance to $\mathbf{1}$ and no resistance to $\mathbf{4 5}$ (Table 5).

\section{Physicochemical and in vitro ADME}

To assess drug-like properties of chemotype 4, selected analogues were tested in various physicochemical and in vitro ADME studies (Table 7). First, aqueous stability and solubility were evaluated in Dulbecco's Phosphate-Buffered Saline (DPBS). The selected compounds showed excellent stability and good solubility in DPBS with the exception of $\mathbf{4 5}$ showed a lower but still acceptable solubility. Second, the selected compounds were found to be stable in both human and mouse plasma. Third, all compounds showed a higher protein binding in human plasma (98-99\%) than that in mouse plasma (71-96\%). Finally, while the selected compounds exhibited excellent phase I stability in both human and mouse liver microsomes, they were much less stable against phase II glucuronidation in microsomes from both species (Table 7). Taken together, although the selected compounds still need improvement 
in their phase II glucuronidation stability, they possess largely favorable physicochemical and in vitro ADME properties. It is also noteworthy that our chemotype has a significant smaller size (MW for $\mathbf{4 5}=322$; the MW for drugs $\mathbf{1 - 3}$ is in the range of 419-448) while supporting antiviral activities comparable to $\mathbf{1}$ (Table 4). The small size of our chemotype would allow significant room for further optimization.

\section{Conclusion}

A new HPD subtype was designed and synthesized. Key to the design is the introduction of an $N$-benzyl-5-carboxamide moiety to mimic the second-generation INSTI 3. SAR revealed the requirement of an alkylamino group at C6 and an $\mathrm{N}$-benzyl group on the C5 carboxamide for INST inhibition and antiviral activity. Numerous analogues demonstrated low nanomolar potencies against HIV-1 in cell culture and low nanomolar biochemical inhibition against INST. Molecular modeling showed that the representative inhibitor $\mathbf{4 5}$ binds to IN with a mode similar to $\mathbf{3}$. Antiviral testing of $\mathbf{4 5}$ against a panel of raltegravirresistant HIV-1 clones showed a resistance profile similar to that of $\mathbf{3}$. However, analogues of our new chemotype also potently inhibited the RNase $\mathrm{H}$ function of RT, suggesting that the antiviral potency could be due to dual target inhibition. in vitro ADME assays also demonstrated largely favorable physicochemical properties for the new chemotype.

\section{Experimental}

\section{Chemistry}

General Procedures-All commercial chemicals were used as supplied unless otherwise indicated. Dry solvents were either purchased (dioxane and $\mathrm{MeOH}$ ) or dispensed under argon from an anhydrous solvent system with two packed columns of neutral alumina or molecular sieves. Flash chromatography was performed on a Teledyne Combiflash RF-200 with RediSep columns (silica) and indicated mobile phase. All moisture sensitive reactions were performed under an inert atmosphere of ultra-pure argon with oven-dried glassware. ${ }^{1} \mathrm{H}$ and ${ }^{13} \mathrm{C}$ NMR spectra were recorded on a Varian $600 \mathrm{MHz}$ spectrometer. Mass data were acquired on an Agilent TOF II TOS/MS spectrometer capable of ESI and APCI ion sources. Analysis of sample purity was performed on a Varian Prepstar SD-1 HPLC system with a Phenomenex Gemini, 5 micron C18 column $(250 \mathrm{~mm} \times 4.6 \mathrm{~mm})$. HPLC conditions: solvent $\mathrm{A}=\mathrm{H}_{2} \mathrm{O}$ containing $0.1 \%$ TFA, solvent $\mathrm{B}=\mathrm{MeCN}$; flow rate $=$ $1.0 \mathrm{~mL} / \mathrm{min}$; compounds were eluted with a gradient of $20 \% \mathrm{MeCN} / \mathrm{H}_{2} \mathrm{O}$ to $100 \% \mathrm{MeCN}$ for $30 \mathrm{~min}$. Purity was determined by total absorbance at $254 \mathrm{~nm}$. All tested compounds have a purity $\geq 96 \%$.

\section{General method of 3-O-benzyl deprotection for the synthesis of final compounds 24-55}

Method A: catalytic hydrogenation-To a solution of $100 \mathrm{mg}$ of 3-(benzyloxy)pyrimidine-2,4(1H,3H)-dione intermediate $(\mathbf{1 0}, \mathbf{1 2}, \mathbf{1 4}-\mathbf{1 6}, \mathbf{2 0})$ in $7.0 \mathrm{~mL} \mathrm{MeOH}$ was added $20 \mathrm{mg}$ Pearlman's catalyst (Pd-C, 20\%). The reaction mixture was degassed using vacuum and refilling with $\mathrm{H}_{2}$ (40-50 psi) for three times. Then keep the reaction mixture being shaken under $\mathrm{H}_{2}$ (40-50 psi) atmosphere for appropriate time. The reaction was monitored by both TLC and LC-MS. The reaction mixture was filtered through a short celite column 
and then removed the solvent. Trituration with $\mathrm{MeOH}$, ethyl acetate and DCM provided desired final compounds $\mathbf{2 4 - 5 5}$ in $82-97 \%$ yield.

Method B: acid hydrolysis-To a microwave reaction vessel were added $0.26 \mathrm{mmol}$ of a 3-(benzyloxy)-pyrimidine-2,4(1H,3H)-dione intermediate $(\mathbf{1 0}, \mathbf{1 2}, \mathbf{1 4 - 1 6}, \mathbf{2 0})$ and $6 \sim 10$ $\mathrm{mL}$ of TFA. The reaction vessel was irradiated at $120{ }^{\circ} \mathrm{C}$ for the appropriate time. The reaction was monitored by both TLC and LC-MS. The reaction mixture was transferred to a round-bottom flask to remove the solvent under reduced pressure. Then the residue was purified by flash chromatography on $\mathrm{C} 18$ reverse phase column $\left(\mathrm{H}_{2} \mathrm{O}-\mathrm{MeOH}\right)$ or trituration ( $\mathbf{M e O H}$, Ethylacetate and DCM) to provide the desired compounds $\mathbf{2 4 - 5 5}$ as solid in 50$77 \%$.

\section{N-Benzyl-1-(hydroxy)-1,2,3,6-tetrahydro-2,6-dioxo-4-} (phenylamino)pyrimidine-5-carboxamide (24)—White solid, 50\% yield; ${ }^{1} \mathrm{H}$ NMR (600 MHz, DMSO-d6): $\delta 12.72$ (s, 1H), 11.26 (s, 1H), 10.32 (s, 1H), 10.03 (t, $J=2.4,1 \mathrm{H}$ ), 7.43-7.26 (m, 10H), 4.49 (s, 2H); ${ }^{13} \mathrm{C}$ NMR (150 MHz, DMSO- $\left.d 6\right): \delta 167.7,161.6,154.0$, 147.0, 139.3, 135.8, 129.5, 128.4, 127.2, 126.9, 126.5, 124.9, 81.7, 41.9; HRMS-ESI(-) m/z calcd for $\mathrm{C}_{18} \mathrm{H}_{16} \mathrm{~N}_{4} \mathrm{O}_{4} 351.1099[\mathrm{M}-\mathrm{H}]^{-}$, found 351.1103 .

\section{N-Benzyl-1-(hydroxy)-1,2,3,6-tetrahydro-2,6-dioxo-4-}

(biphenylamino)pyrimidine-5-carboxamide (25)—White solid, 77\% yield; ${ }^{1} \mathrm{H}$ NMR (600 MHz, DMSO-d6): $\delta 12.76$ (s, 1H), 11.33 (s, 1H), 10.34 (s, 1H), 10.04 (dd, $J=6.0,4.8$ $1 \mathrm{H}), 7.74-7.20$ (m, 14H), 4.50 (d, $J=5.4,2 \mathrm{H}) ;{ }^{13} \mathrm{C}$ NMR (150 MHz, DMSO-d6): $\delta$ 167.7, 161.6, 154.0, 147.0, 139.3, 138.1, 135.2, 129.0, 128.4, 127.7, 127.5, 127.2, 126.9, 126.5, 125.4, 80.9, 41.9; HRMS-ESI(-) m/z calcd for $\mathrm{C}_{24} \mathrm{H}_{20} \mathrm{~N}_{4} \mathrm{O}_{4} 427.1412[\mathrm{M}-\mathrm{H}]^{-}$, found 427.1414 .

N-Benzyl-1-(hydroxy)-1,2,3,6-tetrahydro-4-(methylamino)-2,6dioxopyrimidine-5-carboxamide (26)—White solid, 39\% yield; ${ }^{1} \mathrm{H}$ NMR $(600 \mathrm{MHz}$, DMSO-d6): $\delta 11.05$ (s, 1H), 10.88 (q, $J=4.8 \mathrm{~Hz}, 1 \mathrm{H}), 10.20(\mathrm{~s}, 1 \mathrm{H}), 9.90(\mathrm{t}, J=6.0 \mathrm{~Hz}$, $1 \mathrm{H}), 7.33-7.22(\mathrm{~m}, 5 \mathrm{H}), 4.43(\mathrm{~d}, J=6.0 \mathrm{~Hz}, 2 \mathrm{H}) ; 2.94(\mathrm{~d}, J=5.4 \mathrm{~Hz}, 3 \mathrm{H}) ;{ }^{13} \mathrm{C}$ NMR $(150$ MHz, DMSO- $d 6): ~ \delta$ 167.7, 161.3, 155.7, 147.3, 139.7, 128.3, 127.1, 126.7, 79.0, 41.7, 28.4; HRMS-ESI(-) m/z calcd for $\mathrm{C}_{13} \mathrm{H}_{14} \mathrm{~N}_{4} \mathrm{O}_{4} 289.0942[\mathrm{M}-\mathrm{H}]^{-}$, found 289.0939.

\section{N-(3-Fluorobenzyl)-1-(hydroxy)-1,2,3,6-tetrahydro-4-(methylamino)-2,6-} dioxopyrimidine-5-carboxamide (27)—White solid, $20 \%$ yield; ${ }^{1} \mathrm{H}$ NMR $(600 \mathrm{MHz}$, DMSO-d6): $\delta 11.05$ (s, 1H), 10.80 (q, $J=4.8 \mathrm{~Hz}, 1 \mathrm{H}), 10.18(\mathrm{~s}, 1 \mathrm{H}), 9.94(\mathrm{t}, J=6.0 \mathrm{~Hz}$, $1 \mathrm{H}), 7.35$ (ddd, $J=7.8,7.2,6.0 \mathrm{~Hz}, 1 \mathrm{H}), 7.11$ (d, $J=7.2 \mathrm{~Hz}, 1 \mathrm{H}), 7.06$ (m, 2H), 4.44 (d, $J=$ $6.0 \mathrm{~Hz}, 2 \mathrm{H}) ; 2.93(\mathrm{~d}, J=4.2 \mathrm{~Hz}, 3 \mathrm{H}) ;{ }^{13} \mathrm{C}$ NMR (150 MHz, DMSO-d6): $\delta 167.8,162.2(\mathrm{~d}$, $\left.J_{\mathrm{CF}}=241.5 \mathrm{~Hz}\right), 161.3,155.9,147.4,143.0\left(\mathrm{~d}, J_{\mathrm{CF}}=6.9 \mathrm{~Hz}\right), 130.2\left(\mathrm{~d}, J_{\mathrm{CF}}=8.1 \mathrm{~Hz}\right), 123.1$ $\left(\mathrm{d}, J_{\mathrm{CF}}=2.4 \mathrm{~Hz}\right), 113.7\left(\mathrm{~d}, J_{\mathrm{CF}}=21.9 \mathrm{~Hz}\right), 113.4\left(\mathrm{~d}, J_{\mathrm{CF}}=20.0 \mathrm{~Hz}\right), 79.0,41.2,28.4$; HRMS-ESI(-) m/z calcd for $\mathrm{C}_{13} \mathrm{H}_{13} \mathrm{FN}_{4} \mathrm{O}_{4} 307.0848[\mathrm{M}-\mathrm{H}]^{-}$, found 307.0852 .

\section{N-(4-Fluorobenzyl)-1-(hydroxy)-1,2,3,6-tetrahydro-4-(methylamino)-2,6-} dioxopyrimidine-5-carboxamide (28)—White solid, $20 \%$ yield; ${ }^{1} \mathrm{H}$ NMR $(600 \mathrm{MHz}$, DMSO-d6): $\delta 11.05$ (s, 1H), 10.86 (q, $J=5.4 \mathrm{~Hz}, 1 \mathrm{H}), 10.19$ (s, 1H), 9.87 (t, $J=6.0 \mathrm{~Hz}$, 
1H), 7.31 (dd, $J=8.4,5.4 \mathrm{~Hz}, 2 \mathrm{H}), 7.14(\mathrm{t}, J=9.0 \mathrm{~Hz}, 2 \mathrm{H}), 4.41$ (d, $J=5.4 \mathrm{~Hz}, 2 \mathrm{H}) ; 2.94$ (d, $J=4.8 \mathrm{~Hz}, 3 \mathrm{H}) ;{ }^{13} \mathrm{C}$ NMR (150 MHz, DMSO- $\left.d \sigma\right): ~ \delta 167.7,163.7,161.1\left(\mathrm{~d}, J_{\mathrm{CF}}=241.5\right.$ $\mathrm{Hz}), 161.2,155.7,147.3,136.0\left(\mathrm{~d}, J_{\mathrm{CF}}=2.4 \mathrm{~Hz}\right), 129.1\left(\mathrm{~d}, J_{\mathrm{CF}}=8.1 \mathrm{~Hz}\right), 115.0\left(\mathrm{~d}, J_{\mathrm{CF}}=\right.$ $20.7 \mathrm{~Hz}$ ), 79.0, 40.9, 28.4; HRMS-ESI(-) m/z calcd for $\mathrm{C}_{13} \mathrm{H}_{13} \mathrm{FN}_{4} \mathrm{O}_{4} 307.0848[\mathrm{M}-\mathrm{H}]^{-}$, found 307.0850 .

\section{$\mathrm{N}$-(3-Chloro-2-fluorobenzyl)-1-(hydroxy)-1,2,3,6-tetrahydro-4-} (methylamino)-2,6-dioxopyrimidine-5-carboxamide (29)—White solid, $90 \%$ yield; ${ }^{1} \mathrm{H}$ NMR (600 MHz, DMSO- $d 6$ ): $\delta 11.06(\mathrm{~s}, 1 \mathrm{H}), 10.76(\mathrm{q}, J=4.2 \mathrm{~Hz}, 1 \mathrm{H}), 10.22$ (s, $1 \mathrm{H}), 9.95(\mathrm{t}, J=6.0 \mathrm{~Hz}, 1 \mathrm{H}), 7.47(\mathrm{t}, J=7.2 \mathrm{~Hz}, 1 \mathrm{H}), 7.27(\mathrm{dd}, J=7.8,6.0 \mathrm{~Hz}, 1 \mathrm{H}), 7.18(\mathrm{t}$, $J=7.8 \mathrm{~Hz}, 1 \mathrm{H}), 4.50(\mathrm{~d}, J=4.8 \mathrm{~Hz}, 2 \mathrm{H}), 2.93(\mathrm{~d}, J=4.2 \mathrm{~Hz}, 3 \mathrm{H}) ;{ }^{13} \mathrm{C}$ NMR $(150 \mathrm{MHz}$, DMSO- $d 6): \delta 167.8,161.3,155.7,155.2\left(\mathrm{~d}, J_{\mathrm{CF}}=246 \mathrm{~Hz}\right), 147.3,129.0,128.7\left(\mathrm{~d}, J_{\mathrm{CF}}=\right.$ $13.8 \mathrm{~Hz}), 128.2\left(\mathrm{~d}, J_{\mathrm{CF}}=4.5 \mathrm{~Hz}\right), 125.2\left(\mathrm{~d}, J_{\mathrm{CF}}=4.7 \mathrm{~Hz}\right), 119.4\left(\mathrm{~d}, J_{\mathrm{CF}}=17.3 \mathrm{~Hz}\right), 79.0$, $35.9\left(\mathrm{~d}, J_{\mathrm{CF}}=3.5 \mathrm{~Hz}\right), 28.4 ;$ HRMS-ESI(-) m/z calcd for $\mathrm{C}_{13} \mathrm{H}_{12} \mathrm{ClFN}_{4} \mathrm{O}_{4} 341.0458[\mathrm{M}$ $-\mathrm{H}]^{-}$, found 341.0462 .

\section{N-Benzyl-1-(hydroxy)-4-(ethylamino)-1,2,3,6-tetrahydro-2,6-} dioxopyrimidine-5-carboxamide (30)—White solid, $42 \%$ yield; ${ }^{1} \mathrm{H}$ NMR (600 MHz, DMSO-d6): $\delta 11.11(\mathrm{~s}, 1 \mathrm{H}), 11.04(\mathrm{t}, J=5.4 \mathrm{~Hz}, 1 \mathrm{H}), 10.20(\mathrm{~s}, 1 \mathrm{H}), 9.93(\mathrm{t}, J=6.0 \mathrm{~Hz}, 1 \mathrm{H})$, 7.34-7.24 (m, 5H), $4.43(\mathrm{~d}, J=6.0 \mathrm{~Hz}, 2 \mathrm{H}) ; 3.36(\mathrm{td}, J=6.6,6.0 \mathrm{~Hz}, 2 \mathrm{H}), 1.14(\mathrm{t}, J=6.6$ $\mathrm{Hz}, 3 \mathrm{H}) ;{ }^{13} \mathrm{C}$ NMR (150 MHz, DMSO-d6): $\delta$ 167.8, 161.4, 154.7, 147.3, 139.6, 128.4, 127.1, 126.8, 78.8, 41.7, 36.0, 14.4; HRMS-ESI(-) m/z calcd for $\mathrm{C}_{14} \mathrm{H}_{16} \mathrm{~N}_{4} \mathrm{O}_{4} 303.1099[\mathrm{M}$ $-\mathrm{H}]^{-}$, found 303.1098 .

\section{N-Benzyl-1-(hydroxy)-1,2,3,6-tetrahydro-2,6-dioxo-4-} (propylamino)pyrimidine-5-carboxamide (31)—White solid, $79 \%$ yield; ${ }^{1} \mathrm{H}$ NMR (600 MHz, DMSO-d6): $\delta 11.16$ (s, 1H), 11.13 (s, 1H), 10.20 (s, 1H), $9.94(\mathrm{~s}, 1 \mathrm{H}), 7.32-7.24$ $(\mathrm{m}, 5 \mathrm{H}), 4.44(\mathrm{~d}, J=4.8 \mathrm{~Hz}, 2 \mathrm{H}) ; 3.30(\mathrm{~m}, 2 \mathrm{H}), 1.54(\mathrm{~m}, 2 \mathrm{H}), 0.91(\mathrm{t}, J=6.6 \mathrm{~Hz}, 3 \mathrm{H}) ;{ }^{13} \mathrm{C}$ NMR (150 MHz, DMSO-d6): $\delta$ 167.9, 161.4, 154.9, 147.3, 139.6, 128.4, 127.1, 126.8, 78.8, 42.6, 41.7, 22.1, 11.0; HRMS-ESI(-) m/z calcd for $\mathrm{C}_{15} \mathrm{H}_{18} \mathrm{~N}_{4} \mathrm{O}_{4} 317.1255[\mathrm{M}-\mathrm{H}]^{-}$, found 317.1253 .

\section{N-(3-Chloro-2-fluorobenzyl)-1-(hydroxy)-1,2,3,6-tetrahydro-2,6-dioxo-4-} (propylamino)pyrimidine-5-carboxamide (32)—White solid, $73 \%$ yield; ${ }^{1} \mathrm{H}$ NMR (600 MHz, DMSO-d6): $\delta 11.15$ (s, 1H), 11.02 (s, 1H), 10.23 (s, 1H), 9.99 (s, 1H), 7.47-7.18 $(\mathrm{m}, 3 \mathrm{H}), 4.51(\mathrm{~d}, J=4.2 \mathrm{~Hz}, 2 \mathrm{H}), 3.30(\mathrm{~m}, 2 \mathrm{H}), 1.52(\mathrm{~m}, 2 \mathrm{H}), 0.89$ (dd, $J=7.8,6.0 \mathrm{~Hz}$, $3 \mathrm{H}) ;{ }^{13} \mathrm{C}$ NMR (150 MHz, DMSO-d6): $\delta 168.0,161.4,155.2\left(\mathrm{~d}, J_{\mathrm{CF}}=246 \mathrm{~Hz}\right), 154.9$, $147.3,129.1,128.6\left(\mathrm{~d}, J_{\mathrm{CF}}=14.9 \mathrm{~Hz}\right), 128.2\left(\mathrm{~d}, J_{\mathrm{CF}}=4.5 \mathrm{~Hz}\right), 125.3\left(\mathrm{~d}, J_{\mathrm{CF}}=3.5 \mathrm{~Hz}\right)$, $119.5\left(\mathrm{~d}, J_{\mathrm{CF}}=17.3 \mathrm{~Hz}\right), 78.8,42.6,35.9\left(\mathrm{~d}, J_{\mathrm{CF}}=3.5 \mathrm{~Hz}\right), 22.0,11.0 ;$ HRMS-ESI(-) m/z calcd for $\mathrm{C}_{15} \mathrm{H}_{16} \mathrm{ClFN}_{4} \mathrm{O}_{4} 369.0771[\mathrm{M}-\mathrm{H}]-$, found 369.0768 .

\section{N-Benzyl-1-(hydroxy)-1,2,3,6-tetrahydro-4-(isopropylamino)-2,6-} dioxopyrimidine-5-carboxamide (33)—White solid, 92\% yield; ${ }^{1} \mathrm{H}$ NMR (600 MHz, DMSO-d6): $\delta 11.18$ (d, $J=8.4 \mathrm{~Hz} 1 \mathrm{H}), 11.14$ (s, 1H), 10.21 (s, 1H), 9.96 (d, $J=5.4 \mathrm{~Hz} 1 \mathrm{H}$ ), 7.34-7.23 (m, 5H), $4.43(\mathrm{~d}, J=5.4 \mathrm{~Hz}, 2 \mathrm{H}) ; 4.08(\mathrm{~m}, 1 \mathrm{H}), 1.16(\mathrm{~d}, J=6.0 \mathrm{~Hz}, 6 \mathrm{H}) ;{ }^{13} \mathrm{C}$ NMR (150 MHz, DMSO-d6): $\delta$ 167.9, 161.4, 153.8, 147.3, 139.6, 128.4, 127.1, 126.8, 78.7, 
42.6, 41.7, 22.8; HRMS-ESI(-) m/z calcd for $\mathrm{C}_{15} \mathrm{H}_{18} \mathrm{~N}_{4} \mathrm{O}_{4} 317.1255[\mathrm{M}-\mathrm{H}]^{-}$, found 317.1261 .

N-Benzyl-1-(hydroxy)-4-(cyclopropylamino)-1,2,3,6-tetrahydro-2,6dioxopyrimidine-5-carboxamide (34)-White solid, 82\% yield; ${ }^{1} \mathrm{H}$ NMR (600 MHz, DMSO-d6): $\delta 11.17$ (s, $1 \mathrm{H}), 11.01(\mathrm{~s}, 1 \mathrm{H}), 10.24(\mathrm{~s}, 1 \mathrm{H}), 9.87(\mathrm{t}, J=6.0 \mathrm{~Hz} 1 \mathrm{H}), 7.31-7.21$ $(\mathrm{m}, 5 \mathrm{H}), 4.39(\mathrm{~d}, J=5.4 \mathrm{~Hz}, 2 \mathrm{H}) ; 2.71(\mathrm{~m}, 1 \mathrm{H}), 0.83(\mathrm{~m}, 2 \mathrm{H}), 0.59(\mathrm{~m}, 2 \mathrm{H}) ;{ }^{13} \mathrm{C}$ NMR $(150$ MHz, DMSO-d6): $\delta$ 167.6, 161.2, 156.5, 147.1, 139.5, 128.4, 127.1, 126.8, 79.2, 41.7, 23.0, 7.7; HRMS-ESI(-) m/z calcd for $\mathrm{C}_{15} \mathrm{H}_{16} \mathrm{~N}_{4} \mathrm{O}_{4} 315.1099$ [M-H] $]^{-}$, found 315.1101.

4-(Tert-butylamino)-N-benzyl-1-(hydroxy)-1,2,3,6-tetrahydro-2,6dioxopyrimidine-5-carboxamide (35)—White solid, 59\% yield; ${ }^{1} \mathrm{H}$ NMR $(600 \mathrm{MHz}$, DMSO-d6): $\delta 11.82$ (s, 1H), 10.24 (s, 1H), 10.10 (t, $J=6.0 \mathrm{~Hz}, 1 \mathrm{H}), 10.03$ (brs, 1H), 7.337.25 (m, 5H), 4.43 (d, $J=5.4 \mathrm{~Hz}, 2 \mathrm{H}), 1.41$ (s, 9H); ${ }^{13} \mathrm{C}$ NMR (150 MHz, DMSO-d6): $\delta$ 168.1, 161.4, 153.7, 146.8, 139.5, 128.4, 127.1, 126.8, 78.9, 52.0, 41.7, 29.0; HRMS-ESI(-) $\mathrm{m} / \mathrm{z}$ calcd for $\mathrm{C}_{16} \mathrm{H}_{20} \mathrm{~N}_{4} \mathrm{O}_{4} 331.1412[\mathrm{M}-\mathrm{H}]^{-}$, found 331.1416.

\section{4-Amino-N-benzyl-1-(hydroxy)-1,2,3,6-tetrahydro-2,6-dioxopyrimidine-5-} carboxamide (46)—White solid, 89\% yield; ${ }^{1} \mathrm{H}$ NMR (600 MHz, DMSO-d6): $\delta 11.19$ (brs, 1H), 10.13 (brs, 1H), 9.78 (s, 1H), 9.68 (s, 1H), 7.32-7.24 (m, 4H), 6.88 (brs, 1H), 4.43 (d, $J=4.8 \mathrm{~Hz}, 2 \mathrm{H}) ;{ }^{13} \mathrm{C}$ NMR (150 MHz, DMSO- $\left.d \sigma\right): \delta 167.1,161.8,156.4,147.5,139.7$, 128.3, 127.1, 126.7, 79.8, 41.6; HRMS-ESI(-) m/z calcd for $\mathrm{C}_{12} \mathrm{H}_{12} \mathrm{~N}_{4} \mathrm{O}_{4} 275.0786$ [M $-\mathrm{H}]^{-}$, found 275.0785 .

N-(3-Fluorobenzyl)-1-(hydroxy)-4-(ethylamino)-1,2,3,6-tetrahydro-2,6dioxopyrimidine-5-carboxamide (47)—White solid, 76\% yield; ${ }^{1} \mathrm{H}$ NMR $(600 \mathrm{MHz}$, DMSO-d6): $\delta 11.11$ (s, 1H), 11.98 (t, $J=4.8 \mathrm{~Hz}, 1 \mathrm{H}), 10.22(\mathrm{~s}, 1 \mathrm{H}), 9.97(\mathrm{t}, J=6.0 \mathrm{~Hz}, 1 \mathrm{H})$, $7.36(\mathrm{q}, 7.2 \mathrm{~Hz}, 1 \mathrm{H}), 7.11(\mathrm{~d}, J=7.2 \mathrm{~Hz}, 1 \mathrm{H}), 7.06(\mathrm{~m}, 2 \mathrm{H}), 4.45(\mathrm{~d}, J=6.0 \mathrm{~Hz}, 2 \mathrm{H}) ; 3.36$ $(\mathrm{td}, J=7.2,6.6 \mathrm{~Hz}, 2 \mathrm{H}), 1.14(\mathrm{t}, J=7.2 \mathrm{~Hz}, 3 \mathrm{H}) ;{ }^{13} \mathrm{C}$ NMR (150 MHz, DMSO-d6): $\delta 167.9$, $161.5\left(\mathrm{~d}, J_{\mathrm{CF}}=241.5 \mathrm{~Hz}\right), 161.4,154.7,147.3,142.9\left(\mathrm{~d}, J_{\mathrm{CF}}=6.9 \mathrm{~Hz}\right), 130.3\left(\mathrm{~d}, J_{\mathrm{CF}}=8.0\right.$ $\mathrm{Hz}), 123.0,113.7\left(\mathrm{~d}, J_{\mathrm{CF}}=21.8 \mathrm{~Hz}\right), 113.5\left(\mathrm{~d}, J_{\mathrm{CF}}=20.7 \mathrm{~Hz}\right), 78.8,41.2,36.1,14.4$; HRMS-ESI(-) m/z calcd for $\mathrm{C}_{14} \mathrm{H}_{15} \mathrm{FN}_{4} \mathrm{O}_{4} 321.1005[\mathrm{M}-\mathrm{H}]^{-}$, found 321.1005.

\section{N-(4-Fluorobenzyl)-1-(hydroxy)-4-(ethylamino)-1,2,3,6-tetrahydro-2,6-} dioxopyrimidine-5-carboxamide (38)—White solid, 33\% yield; ${ }^{1} \mathrm{H}$ NMR $(600 \mathrm{MHz}$, DMSO-d6): $\delta 11.10(\mathrm{~s}, 1 \mathrm{H}), 11.01(\mathrm{t}, J=5.4 \mathrm{~Hz}, 1 \mathrm{H}), 10.20(\mathrm{~s}, 1 \mathrm{H}), 9.93(\mathrm{t}, J=6.0 \mathrm{~Hz}, 1 \mathrm{H})$, 7.31 (dd, $J=8.4,5.4 \mathrm{~Hz}, 2 \mathrm{H}), 7.14(\mathrm{t}, J=9.0 \mathrm{~Hz}, 2 \mathrm{H}), 4.41(\mathrm{~d}, J=5.4 \mathrm{~Hz}, 2 \mathrm{H}), 3.36$ (td, $J=$ 7.2, $6.6 \mathrm{~Hz}, 2 \mathrm{H}), 1.14(\mathrm{t}, J=7.2 \mathrm{~Hz}, 3 \mathrm{H}) ;{ }^{13} \mathrm{C}$ NMR (150 MHz, DMSO-d6): $\delta 167.8,161.3$, $161.1\left(\mathrm{~d}, J_{\mathrm{CF}}=240.3 \mathrm{~Hz}\right), 154.7,147.3,135.9\left(\mathrm{~d}, J_{\mathrm{CF}}=2.3 \mathrm{~Hz}\right), 129.1\left(\mathrm{~d}, J_{\mathrm{CF}}=8.0 \mathrm{~Hz}\right)$, $115.0\left(\mathrm{~d}, J_{\mathrm{CF}}=21.9 \mathrm{~Hz}\right), 78.8,40.9,36.0,14.4$; HRMS-ESI(-) $\mathrm{m} / \mathrm{z}$ calcd for $\mathrm{C}_{14} \mathrm{H}_{15} \mathrm{FN}_{4} \mathrm{O}_{4}$ $321.1005[\mathrm{M}-\mathrm{H}]^{-}$, found 321.1003 .

N-(2,5-difluorobenzyl)-1-(hydroxy)-4-(ethylamino)-1,2,3,6-tetrahydro-2,6dioxopyrimidine-5-carboxamide (39)—White solid, $32 \%$ yield; ${ }^{1} \mathrm{H}$ NMR $(600 \mathrm{MHz}$, DMSO-dб): $\delta 11.12$ (s, 1H), 11.90 (t, $J=5.4 \mathrm{~Hz}, 1 \mathrm{H}), 10.23$ (s, 1H), 9.96 (t, $J=6.0 \mathrm{~Hz}, 1 \mathrm{H}$ ), 
7.25-7.07 (m, 3H), $4.45(\mathrm{~d}, J=5.4 \mathrm{~Hz}, 2 \mathrm{H}) ; 3.36(\mathrm{td}, J=7.2,6.6 \mathrm{~Hz}, 2 \mathrm{H}), 1.13(\mathrm{t}, J=7.2$ $\mathrm{Hz}, 3 \mathrm{H}$ ); HRMS-ESI(-) m/z calcd for $\mathrm{C}_{14} \mathrm{H}_{14} \mathrm{~F}_{2} \mathrm{~N}_{4} \mathrm{O}_{4} 339.091[\mathrm{M}-\mathrm{H}]^{-}$, found 339.0911.

N-(2,4-difluorobenzyl)-1-(hydroxy)-4-(ethylamino)-1,2,3,6-tetrahydro-2,6dioxopyrimidine-5-carboxamide (40)—White solid, $26 \%$ yield; ${ }^{1} \mathrm{H}$ NMR $(600 \mathrm{MHz}$, DMSO-d6): $\delta 11.11$ (s, 1H), $10.91(\mathrm{t}, J=5.4 \mathrm{~Hz}, 1 \mathrm{H}), 10.19$ (s, $1 \mathrm{H}), 9.94(\mathrm{t}, J=6.0 \mathrm{~Hz}, 1 \mathrm{H})$, 7.36-7.04 (m, 3H), $4.43(\mathrm{~d}, J=6.0 \mathrm{~Hz}, 2 \mathrm{H}) ; 3.35(\mathrm{td}, J=7.2,6.6 \mathrm{~Hz}, 2 \mathrm{H}), 1.13(\mathrm{t}, J=7.2$ $\mathrm{Hz}, 3 \mathrm{H}$ ); HRMS-ESI(-) m/z calcd for $\mathrm{C}_{14} \mathrm{H}_{14} \mathrm{~F}_{2} \mathrm{~N}_{4} \mathrm{O}_{4} 339.091[\mathrm{M}-\mathrm{H}]^{-}$, found 339.0915 .

N-(3,5-difluorobenzyl)-1-(hydroxy)-4-(ethylamino)-1,2,3,6-tetrahydro-2,6dioxopyrimidine-5-carboxamide (41)—White solid, 61\% yield; ${ }^{1} \mathrm{H}$ NMR $(600 \mathrm{MHz}$, DMSO-d6): $\delta 11.12$ (s, 1H), 10.92 (s, 1H), 10.23 (s, 1H), 9.99 (t, $J=5.4 \mathrm{~Hz}, 1 \mathrm{H}), 7.09-6.96$ $(\mathrm{m}, 3 \mathrm{H}), 4.45(\mathrm{~d}, J=5.4 \mathrm{~Hz}, 2 \mathrm{H}) ; 3.36(\mathrm{~m}, 2 \mathrm{H}), 1.14(\mathrm{dd}, J=7.2,6.6 \mathrm{~Hz}, 3 \mathrm{H})$; HRMSESI(-) $\mathrm{m} / \mathrm{z}$ calcd for $\mathrm{C}_{14} \mathrm{H}_{14} \mathrm{~F}_{2} \mathrm{~N}_{4} \mathrm{O}_{4} 339.091[\mathrm{M}-\mathrm{H}]^{-}$, found 339.0914 .

\section{N-(3,4-difluorobenzyl)-1-(hydroxy)-4-(ethylamino)-1,2,3,6-tetrahydro-2,6-} dioxopyrimidine-5-carboxamide (42)—White solid, 56\% yield; ${ }^{1} \mathrm{H}$ NMR $(600 \mathrm{MHz}$, DMSO-d6): $\delta 11.11(\mathrm{~s}, 1 \mathrm{H}), 10.96(\mathrm{~s}, 1 \mathrm{H}), 10.21(\mathrm{~s}, 1 \mathrm{H}), 9.96(\mathrm{~s}, 1 \mathrm{H}), 7.38-7.16(\mathrm{~m}, 3 \mathrm{H})$, $4.41(\mathrm{~d}, J=3.6 \mathrm{~Hz}, 2 \mathrm{H}) ; 3.36(\mathrm{~m}, 2 \mathrm{H}), 1.14$ (dd, $J=7.2 .4 .8 \mathrm{~Hz}, 3 \mathrm{H}) ; \operatorname{HRMS}-E S I(-) \mathrm{m} / \mathrm{z}$ calcd for $\mathrm{C}_{14} \mathrm{H}_{14} \mathrm{~F}_{2} \mathrm{~N}_{4} \mathrm{O}_{4} 339.091[\mathrm{M}-\mathrm{H}]^{-}$, found 339.0915 .

$\mathrm{N}$-(2-Chloro-5-fluorobenzyl)-1-(hydroxy)-4-(ethylamino)-1,2,3,6tetrahydro-2,6-dioxopyrimidine-5-carboxamide (43)—White solid, $20 \%$ yield; ${ }^{1} \mathrm{H}$ NMR (600 MHz, DMSO-d6): $\delta 11.13$ (s, 1H), 10.88 (dd, $J=5.4,4.2 \mathrm{~Hz}, 1 \mathrm{H}), 10.24$ (s, 1H), $10.03(\mathrm{t}, J=6.0 \mathrm{~Hz}, 1 \mathrm{H}), 7.50(\mathrm{dd}, J=9.0,5.4 \mathrm{~Hz}, 1 \mathrm{H}), 7.16(\mathrm{td}, J=8.4,3.0 \mathrm{~Hz}, 1 \mathrm{H}), 7.09$ (dd, $J=9.6,2.4 \mathrm{~Hz}, 1 \mathrm{H}), 4.47$ (d, $J=6.0 \mathrm{~Hz}, 2 \mathrm{H}), 3.35(\mathrm{td}, J=7.2,6.6 \mathrm{~Hz}, 2 \mathrm{H}), 1.13(\mathrm{t}, J=$ $7.2 \mathrm{~Hz}, 3 \mathrm{H}) ;{ }^{13} \mathrm{C}$ NMR (150 MHz, DMSO- $\left.d 6\right): \delta 168.0,161.4,160.8\left(\mathrm{~d}, J_{\mathrm{CF}}=242.7 \mathrm{~Hz}\right.$ ), $154.7,147.3,139.5\left(\mathrm{~d}, J_{\mathrm{CF}}=6.9 \mathrm{~Hz}\right), 130.9\left(\mathrm{~d}, J_{\mathrm{CF}}=8.1 \mathrm{~Hz}\right), 127.2\left(\mathrm{~d}, J_{\mathrm{CF}}=2.3 \mathrm{~Hz}\right), 116.2$ $\left(\mathrm{d}, J_{\mathrm{CF}}=24.2 \mathrm{~Hz}\right), 115.4\left(\mathrm{~d}, J_{\mathrm{CF}}=23.1 \mathrm{~Hz}\right), 78.8,39.8,36.1,14.3$; HRMS-ESI $(-) \mathrm{m} / \mathrm{z}$ calcd for $\mathrm{C}_{14} \mathrm{H}_{14} \mathrm{ClFN}_{4} \mathrm{O}_{4} 355.0615[\mathrm{M}-\mathrm{H}]-$, found 355.0618 .

\section{N-(4-Chloro-3-fluorobenzyl)-1-(hydroxy)-4-(ethylamino)-1,2,3,6-} tetrahydro-2,6-dioxopyrimidine-5-carboxamide (44)—White solid, $51 \%$ yield; ${ }^{1} \mathrm{H}$ NMR (600 MHz, DMSO- $d 6)$ : $\delta 11.12(\mathrm{~s}, 1 \mathrm{H}), 10.94(\mathrm{t}, J=5.4 \mathrm{~Hz}, 1 \mathrm{H}), 10.22(\mathrm{~s}, 1 \mathrm{H}), 9.98$ $(\mathrm{t}, J=6.0 \mathrm{~Hz}, 1 \mathrm{H}), 7.53(\mathrm{dd}, J=8.4,7.2 \mathrm{~Hz}, 1 \mathrm{H}), 7.28(\mathrm{~d}, J=10.8 \mathrm{~Hz}, 1 \mathrm{H}), 7.19(\mathrm{~d}, J=8.4$ $\mathrm{Hz}, 1 \mathrm{H}), 4.43(\mathrm{~d}, J=6.0 \mathrm{~Hz}, 2 \mathrm{H}), 3.36(\mathrm{td}, J=7.2,6.6 \mathrm{~Hz}, 2 \mathrm{H}), 1.13(\mathrm{t}, J=7.2 \mathrm{~Hz}, 3 \mathrm{H}) ;{ }^{13} \mathrm{C}$ NMR (150 MHz, DMSO-d6): $\delta$ 168.0, 161.3, $157.0\left(\mathrm{~d}, J_{\mathrm{CF}}=244.8 \mathrm{~Hz}\right), 154.7,147.3,142.0$ $\left(\mathrm{d}, J_{\mathrm{CF}}=5.7 \mathrm{~Hz}\right), 130.4,124.2\left(\mathrm{~d}, J_{\mathrm{CF}}=3.4 \mathrm{~Hz}\right), 117.4\left(\mathrm{~d}, J_{\mathrm{CF}}=17.3 \mathrm{~Hz}\right), 115.4\left(\mathrm{~d}, J_{\mathrm{CF}}=\right.$ $20.7 \mathrm{~Hz}), 78.8,40.8,36.1,14.3$; HRMS-ESI(-) $\mathrm{m} / \mathrm{z}$ calcd for $\mathrm{C}_{14} \mathrm{H}_{14} \mathrm{ClFN}_{4} \mathrm{O}_{4} 355.0615[\mathrm{M}$ $-\mathrm{H}]^{-}$, found 355.0621 .

\section{N-(3-Chloro-2-fluorobenzyl)-1-(hydroxy)-4-(ethylamino)-1,2,3,6-} tetrahydro-2,6-dioxopyrimidine-5-carboxamide (45)—White solid, $90 \%$ yield; ${ }^{1} \mathrm{H}$ NMR (600 MHz, DMSO- $d 6$ ): $\delta 11.12$ (s, 1H), 10.90 (t, $J=5.4 \mathrm{~Hz}, 1 \mathrm{H}), 10.23$ (s, 1H), 9.99 (t, $J=6.0 \mathrm{~Hz}, 1 \mathrm{H}), 7.48(\mathrm{t}, J=7.2 \mathrm{~Hz}, 1 \mathrm{H}), 7.27(\mathrm{dd}, J=7.2 \mathrm{~Hz}, 1 \mathrm{H}), 7.19(\mathrm{t}, J=7.8 \mathrm{~Hz}$, $1 \mathrm{H}), 4.50(\mathrm{~d}, J=4.2 \mathrm{~Hz}, 2 \mathrm{H}), 3.35(\mathrm{td}, J=7.2,6.6 \mathrm{~Hz}, 2 \mathrm{H}), 1.13(\mathrm{t}, J=7.2 \mathrm{~Hz}, 3 \mathrm{H}) ;{ }^{13} \mathrm{C}$ 
NMR (150 MHz, DMSO- $d 6): \delta 167.9,161.4,155.2\left(\mathrm{~d}, J_{\mathrm{CF}}=244.8 \mathrm{~Hz}\right), 154.7,147.3$, $129.1,128.6\left(\mathrm{~d}, J_{\mathrm{CF}}=13.8 \mathrm{~Hz}\right), 128.2\left(\mathrm{~d}, J_{\mathrm{CF}}=3.5 \mathrm{~Hz}\right), 125.2\left(\mathrm{~d}, J_{\mathrm{CF}}=4.5 \mathrm{~Hz}\right), 119.4(\mathrm{~d}$, $\left.J_{\mathrm{CF}}=17.3 \mathrm{~Hz}\right), 78.8,36.0,35.9\left(\mathrm{~d}, J_{\mathrm{CF}}=3.5 \mathrm{~Hz}\right), 14.4$; HRMS-ESI $(-) \mathrm{m} / \mathrm{z}$ calcd for $\mathrm{C}_{14} \mathrm{H}_{14} \mathrm{ClFN}_{4} \mathrm{O}_{4} 355.0615[\mathrm{M}-\mathrm{H}]^{-}$, found 355.0620.

N-(3-Fluoro-4-methylbenzyl)-1-(hydroxy)-4-(ethylamino)-1,2,3,6tetrahydro-2,6-dioxopyrimidine-5-carboxamide (46)—White solid, $37 \%$ yield; ${ }^{1} \mathrm{H}$ NMR (600 MHz, DMSO- $d 6): \delta 11.11(\mathrm{~s}, 1 \mathrm{H}), 11.00(\mathrm{t}, J=5.4 \mathrm{~Hz}, 1 \mathrm{H}), 10.21(\mathrm{~s}, 1 \mathrm{H}), 9.93$ $(\mathrm{t}, J=5.4 \mathrm{~Hz}, 1 \mathrm{H}), 7.22(\mathrm{t}, J=7.8 \mathrm{~Hz}, 1 \mathrm{H}), 7.01(\mathrm{~m}, 2 \mathrm{H}), 4.39(\mathrm{~d}, J=6.0 \mathrm{~Hz}, 2 \mathrm{H}), 3.36(\mathrm{td}$, $J=6.6,6.0 \mathrm{~Hz}, 2 \mathrm{H}), 2.19$ (s, 3H), $1.13(\mathrm{dd}, J=7.2,6.6 \mathrm{~Hz}, 3 \mathrm{H}) ;{ }^{13} \mathrm{C} \mathrm{NMR}(150 \mathrm{MHz}$, DMSO-d6): $\delta 167.9,161.4,160.5\left(\mathrm{~d}, J_{\mathrm{CF}}=239.3 \mathrm{~Hz}\right), 154.7,147.3,139.9\left(\mathrm{~d}, J_{\mathrm{CF}}=6.9 \mathrm{~Hz}\right)$, $131.5\left(\mathrm{~d}, J_{\mathrm{CF}}=5.7 \mathrm{~Hz}\right), 122.8\left(\mathrm{~d}, J_{\mathrm{CF}}=3.5 \mathrm{~Hz}\right), 122.3\left(\mathrm{~d}, J_{\mathrm{CF}}=17.3 \mathrm{~Hz}\right), 113.5\left(\mathrm{~d}, J_{\mathrm{CF}}=\right.$ $21.8 \mathrm{~Hz}), 78.8,41.0,36.0,14.4,13.8\left(\mathrm{~d}, J_{\mathrm{CF}}=3.5 \mathrm{~Hz}\right)$; HRMS-ESI(-) $\mathrm{m} / \mathrm{z}$ calcd for $\mathrm{C}_{15} \mathrm{H}_{17} \mathrm{FN}_{4} \mathrm{O}_{4} 335.1161[\mathrm{M}-\mathrm{H}]^{-}$, found 335.1164 .

1-(Hydroxy)-4-(ethylamino)-1,2,3,6-tetrahydro-2,6-dioxo-Nphenethylpyrimidine-5-carboxamide (47)—White solid, 79\%yield; ${ }^{1} \mathrm{H}$ NMR $(600$ MHz, DMSO-d6): $\delta 11.11$ (t, $J=5.4 \mathrm{~Hz}, 1 \mathrm{H}), 11.06$ (s, 1H), 10.17 (s, 1H), 9.56 (t, $J=5.4$ $\mathrm{Hz}, 1 \mathrm{H}$ ), 7.30-7.19 (m, 5H), 3.45 (ddd, $J=7.2,6.6,6.0 \mathrm{~Hz}, 2 \mathrm{H}$ ); 3.35 (qd, $J=7.2,6.6 \mathrm{~Hz}$, 2H), 2.77 (t, $J=7.2 \mathrm{~Hz}, 3 \mathrm{H}), 1.14$ (t, $J=7.2 \mathrm{~Hz}, 3 \mathrm{H}$ ); ${ }^{13} \mathrm{C}$ NMR (150 MHz, DMSO- $d 6$ ): $\delta$ 167.8, 161.2, 154.6, 147.3, 139.4, 128.6, 128.4, 126.1, 78.9, 39.8, 36.0, 35.3, 14.4; HRMSESI(-) m/z calcd for $\mathrm{C}_{15} \mathrm{H}_{18} \mathrm{~N}_{4} \mathrm{O}_{4} 317.1255[\mathrm{M}-\mathrm{H}]^{-}$, found 317.1257 .

1-(Hydroxy)-4-(ethylamino)-1,2,3,6-tetrahydro-2,6-dioxo-Nphenylpyrimidine-5-carboxamide (48)—White solid, 83\% yield; ${ }^{1} \mathrm{H}$ NMR $(600 \mathrm{MHz}$, DMSO- $d 6$ ): $\delta 11.89$ (s, 1H), 11.26 (s, 1H), 10.89 (t, $J=5.4 \mathrm{~Hz}, 1 \mathrm{H}), 10.38$ (s, 1H), 7.55 (d, $J$ $=7.8 \mathrm{~Hz}, 2 \mathrm{H}), 7.31(\mathrm{dd}, J=7.8,7.2 \mathrm{~Hz}, 2 \mathrm{H}), 7.31(\mathrm{dd}, J=7.8,7.2 \mathrm{~Hz}, 1 \mathrm{H}), 3.42(\mathrm{qd}, J=$ 6.6, $6.0 \mathrm{~Hz}, 2 \mathrm{H}), 1.18(\mathrm{t}, J=6.6 \mathrm{~Hz}, 3 \mathrm{H}) ;{ }^{13} \mathrm{C}$ NMR (150 MHz, DMSO- $\left.d \sigma\right): \delta 166.3,161.8$, 154.9, 147.1, 138.5, 128.9, 123.1, 119.8, 79.2, 36.3, 14.4; HRMS-ESI(-) $\mathrm{m} / \mathrm{z}$ calcd for $\mathrm{C}_{13} \mathrm{H}_{14} \mathrm{~N}_{4} \mathrm{O}_{4} 289.0942[\mathrm{M}-\mathrm{H}]^{-}$, found 289.0942 .

1-(Hydroxy)-1,2,3,6-tetrahydro-2,6-dioxo-4-(phenylamino)pyrimidine-5carboxamide (49)—White solid; ${ }^{1} \mathrm{H}$ NMR (600 MHz, DMSO-d6): $\delta 12.90$ (s, 1H), 11.20 (s, 1H), 10.27 (s, 1H), 8.96 (s, 1H), 7.44-7.27 (m, 5H); ${ }^{13} \mathrm{C}$ NMR (150 MHz, DMSO-d6): $\delta$ 169.9, 161.4, 154.2, 147.0, 135.8, 129.6, 126.4, 124.9, 80.7; HRMS-ESI(-) m/z calcd for $\mathrm{C}_{11} \mathrm{H}_{10} \mathrm{~N}_{4} \mathrm{O}_{4} 261.0629[\mathrm{M}-\mathrm{H}]^{-}$, found 261.0632 .

1-(Hydroxy)-N-(2-chloroethyl)-4-(ethylamino)-1,2,3,6-tetrahydro-2,6dioxopyrimidine-5-carboxamide (50)—White solid, $83 \%$ yield; ${ }^{1} \mathrm{H}$ NMR $(600 \mathrm{MHz}$, DMSO-d6): $\delta 11.11(\mathrm{~s}, 1 \mathrm{H}), 11.98(\mathrm{~s}, 1 \mathrm{H}), 10.22(\mathrm{~s}, 1 \mathrm{H}), 9.78(\mathrm{~s}, 1 \mathrm{H}), 3.68(\mathrm{~m}, 2 \mathrm{H}), 3.55$ (m, 2H), $3.37(\mathrm{~m}, 2 \mathrm{H}), 1.15$ (t, $J=6.6 \mathrm{~Hz}, 3 \mathrm{H}) ;{ }^{13} \mathrm{C}$ NMR (150 MHz, DMSO-d6): $\delta$ 168.0, 161.2, 154.7, 147.3, 78.7, 43.97, 40.1, 36.1, 14.4; HRMS-ESI(-) m/z calcd for $\mathrm{C}_{9} \mathrm{H}_{13} \mathrm{ClN}_{4} \mathrm{O}_{4} 275.0553[\mathrm{M}-\mathrm{H}]^{-}$, found 275.0552.

Phenyl-1-(hydroxy)-4-(ethylamino)-1,2,3,6-tetrahydro-2,6-dioxopyrimidine-5carboxylate (51)—White solid, 92\% yield; ${ }^{1} \mathrm{H}$ NMR (600 MHz, DMSO- $d \sigma$ ): $\delta 11.16$ (s, 
1H), $10.12(\mathrm{~s}, 1 \mathrm{H}), 9.49(\mathrm{~s}, 1 \mathrm{H}), 7.41-7.09(\mathrm{~m}, 5 \mathrm{H}), 3.42(\mathrm{~m}, 2 \mathrm{H}), 1.14(\mathrm{~m}, 3 \mathrm{H}) ;{ }^{13} \mathrm{C}$ NMR (150 MHz, DMSO-d6): $\delta$ 167.2, 157.2, 155.6, 150.7, 147.6, 129.3, 125.3, 122.1, 78.2, 36.5, 14.2; HRMS-ESI(-) m/z calcd for $\mathrm{C}_{13} \mathrm{H}_{13} \mathrm{~N}_{3} \mathrm{O}_{5} 290.0782$ [M-H]-, found 290.0784 .

\section{N-(4-Fluorobenzyl)-3-(hydroxy)-6-(ethylamino)-1,2,3,4-tetrahydro-1-} methyl-2,4-dioxopyrimidine-5-carboxamide (52)-White solid, 38\% yield; ${ }^{1} \mathrm{H}$ NMR (600 MHz, DMSO-d6): $\delta 11.89(\mathrm{~s}, 1 \mathrm{H}), 10.29(\mathrm{~s}, 1 \mathrm{H}), 9.93(\mathrm{~s}, 1 \mathrm{H}), 7.32(\mathrm{~m}, 2 \mathrm{H}), 7.14(\mathrm{~m}$, $2 \mathrm{H}), 4.43(\mathrm{~d}, J=4.2 \mathrm{~Hz}, 2 \mathrm{H}), 4.00(\mathrm{~s}, 3 \mathrm{H}), 3.47(\mathrm{~m}, 2 \mathrm{H}), 1.16(\mathrm{dd}, J=6.6,5.4 \mathrm{~Hz}, 3 \mathrm{H}) ;{ }^{13} \mathrm{C}$ NMR (150 MHz, DMSO-d6): $\delta 167.6,161.1\left(\mathrm{~d}, J_{\mathrm{CF}}=240.3 \mathrm{~Hz}\right), 160.5,159.8,154.2$, $135.9,129.1\left(\mathrm{~d}, J_{\mathrm{CF}}=8.1 \mathrm{~Hz}\right), 115.0\left(\mathrm{~d}, J_{\mathrm{CF}}=20.7 \mathrm{~Hz}\right), 84.4,55.6,41.0,35.2,14.8$; HRMS-ESI(-) m/z calcd for $\mathrm{C}_{15} \mathrm{H}_{17} \mathrm{FN}_{4} \mathrm{O}_{4} 335.1161[\mathrm{M}-\mathrm{H}]^{-}$, found 335.1158 .

3-(Benzyloxy)-6-(ethylamino)-N-(4-fluorobenzyl)-N-methyl-2,4-dioxo-1,2,3,4tetrahydropyrimidine-5-carboxamide (53)—White solid, $28 \%$ yield; ${ }^{1} \mathrm{H}$ NMR (600 MHz, DMSO-d6): $\delta 11.25$ (s, 1H), $9.99(\mathrm{~s}, 1 \mathrm{H}), 7.52-7.13(\mathrm{~m}, 9 \mathrm{H}), 4.98(\mathrm{~s}, 2 \mathrm{H}), 4.43(\mathrm{~s}$, 2H), $3.43(\mathrm{~m}, 2 \mathrm{H}), 3.38(\mathrm{~s}, 3 \mathrm{H}), 1.19$ (dd, $J=6.0,5.4 \mathrm{~Hz}, 3 \mathrm{H}) ;{ }^{13} \mathrm{C} \mathrm{NMR}(150 \mathrm{MHz}$, DMSO-d6): $\delta$ 167.5, $161.2\left(\mathrm{~d}, J_{\mathrm{CF}}=240.3 \mathrm{~Hz}\right), 161.0,159.9,148.5,135.7,134.4,129.34$, $129.31\left(\mathrm{~d}, J_{\mathrm{CF}}=8.1 \mathrm{~Hz}\right), 128.8,128.3,115.1\left(\mathrm{~d}, J_{\mathrm{CF}}=20.7 \mathrm{~Hz}\right), 83.4,77.3,41.6,41.2,35.9$, 15.5; HRMS-ESI(-) m/z calcd for $\mathrm{C}_{22} \mathrm{H}_{23} \mathrm{FN}_{4} \mathrm{O}_{4} 425.1631$ [M-H] $]^{-}$, found 425.1631 .

\section{N-Benzyl-1,6-dihydroxy-2,4-dioxo-1,2,3,4-tetrahydropyrimidine-5-} carboxamide (54)—Pale white solid, 53\% yield; 1H NMR (600 MHz, DMSO- $d 6$ ): $\delta$ 11.91 (s, 1H), 10.40 (s, 1H), 9.97 (s, 1H), 7.36-7.29 (m, 5H), 4.57 (brs, 2H); 13C NMR (150 MHz, DMSO-d6): $\delta$ 170.0, 147.6, 137.6, 7, 128.5, 127.41, 127.36, 79.0, 42.8; HRMSESI(-) $\mathrm{m} / \mathrm{z}$ calcd for $\mathrm{C}_{12} \mathrm{H}_{11} \mathrm{~N}_{3} \mathrm{O}_{5} 276.0626[\mathrm{M}-\mathrm{H}]-$, found 276.0628 .

\section{N-(4-Fluorobenzyl)-7-(hydroxy)-2,3,4,6,7,8-hexahydro-6,8-dioxo-1H-} pyrimido[1,6-a]pyrimidine-9-carboxamide (55)—Pale white solid, 96 yield; ${ }^{1} \mathrm{H}$ NMR (600 MHz, DMSO-d6): $\delta 11.52$ (s, 1H), 10.29 (s, 1H), 10.02 (br s, 1H), 7.31-7.13 (m, 4H), $4.42(\mathrm{~d}, J=4.8,2 \mathrm{H}), 3.82(\mathrm{~m}, 2 \mathrm{H}), 3.38(\mathrm{~m}, 2 \mathrm{H}), 1.94(\mathrm{~m}, 2 \mathrm{H}) ;{ }^{13} \mathrm{C}$ NMR $(150 \mathrm{MHz}$, DMSO-d6): $\delta 167.9,161.1\left(\mathrm{~d}, J_{\mathrm{CF}}=241.5 \mathrm{~Hz}\right), 160.4,154.1,147.4,136.0\left(\mathrm{~d}, J_{\mathrm{CF}}=2.3 \mathrm{~Hz}\right)$, $129.1\left(\mathrm{~d}, J_{\mathrm{CF}}=8.1 \mathrm{~Hz}\right), 115.0\left(\mathrm{~d}, J_{\mathrm{CF}}=20.1 \mathrm{~Hz}\right), 78.6,41.0,40.8,38.1,18.8$; HRMSESI(-) $\mathrm{m} / \mathrm{z}$ calcd for $\mathrm{C}_{15} \mathrm{H}_{15} \mathrm{FN}_{4} \mathrm{O}_{4} 333.1005[\mathrm{M}-\mathrm{H}]-$, found 333.1001 .

\section{Biology}

INST assay-HIV integrase was expressed and purified as previously reported. ${ }^{52}$ Inhibition assays were performed using a modified protocol of our reported method. ${ }^{52}$ Briefly, $2.1 \mu \mathrm{L}$ of compound suspended in DMSO was placed in duplicate into a Black 96 well non-binding plate (corning 3991). Compounds were plated in duplicate to a final concentration of $0.13-100 \mu \mathrm{M}$. To each well of the plate $186.9 \mu \mathrm{L}$ of reaction mixture without DNA substrate was added (10 mM HEPES pH 7.5, $10 \%$ glycerol w/v, $10 \mathrm{mM}$ $\mathrm{MnCl} 2,1 \mathrm{mM}$ DTT, $1 \mu \mathrm{M}$ integrase). The enzyme was incubated with inhibitor for $10 \mathrm{~min}$ at $25^{\circ} \mathrm{C}$ after which the reaction was initiated by the addition of $21 \mu \mathrm{L}$ of $500 \mathrm{nM}$ oligo (5' biotin ATGTGGAAAATCTCTAGCA annealed with ACTGCTAGAGATTTTCCACAT 3' Cy5). Reactions were incubated at $37^{\circ} \mathrm{C}$ for $30 \mathrm{~min}$ and then quenched by the addition of 
$5.2 \mu \mathrm{L} 500 \mathrm{mM}$ EDTA. Each reaction was moved $(200 \mu \mathrm{L})$ to a MultiScreen HTS PCR plate (Millipore MSSLBPC10) containing $20 \mu \mathrm{L}$ streptavidin agarose beads (Life Technologies S951) and incubated with shaking for $30 \mathrm{~min}$. A vacuum manifold was used to remove the reaction mixture and the beads were similarly washed 3 times with wash buffer $(.05 \%$ SDS, $1 \mathrm{mM}$ EDTA in PBS). The plates were further washed 3 times with $200 \mu \mathrm{L} 50 \mathrm{mM} \mathrm{NaOH}$ to denature DNA not covalently linked to the biotin modification. For each denaturation step the plate was incubated with shaking at $25^{\circ} \mathrm{C}$ for $5 \mathrm{~min}$ and the $\mathrm{NaOH}$ was removed by centrifugation at $1000 \mathrm{~g}$ for $1 \mathrm{~min}$. The reaction products were eluted from the beads by the addition of $150 \mu \mathrm{L}$ formamide. The plate was incubated at $25^{\circ} \mathrm{C}$ for $10 \mathrm{~min}$ and read directly at 635/675 in a SpectraMax i3 plate reader (Molecular Devices).

Expression and purification of the recombinant IN in Escherichia coli were performed as previously reported ${ }^{39,53}$ with addition of $10 \%$ glycerol to all buffers. Preparation of oligonucleotide substrates has been described. ${ }^{54}$ Integrase reactions were performed in 10 $\mu \mathrm{L}$ with $400 \mathrm{nM}$ of recombinant IN, $20 \mathrm{nM}$ of 5 '-end [ $\left.{ }^{32} \mathrm{P}\right]$-labeled oligonucleotide substrate and inhibitors at various concentrations. Solutions of $10 \%$ DMSO without inhibitors were used as controls. Reactions were incubated at $37{ }^{\circ} \mathrm{C}$ (60 minutes) in buffer containing 50 $\mathrm{mM}$ MOPS, pH 7.2, $7.5 \mathrm{mM} \mathrm{MgCl}_{2}$, and $14.3 \mathrm{mM}$ 2-mercaptoethanol. Reactions were stopped by addition of $10 \mu \mathrm{L}$ of loading dye (10 mM EDTA, 98\% deionized formamide, $0.025 \%$ xylene cyanol and $0.025 \%$ bromophenol blue). Reactions were then subjected to electrophoresis in $20 \%$ polyacrylamide- $7 \mathrm{M}$ urea gels. Gels were dried and reaction products were visualized and quantitated with a Typhoon 8600 (GE Healthcare, Little Chalfont, Buckinghamshire, UK). Densitometric analyses were performed using ImageQuant from Molecular Dynamics Inc. The concentrations at which enzyme activity was reduced by $50 \%\left(\mathrm{IC}_{50}\right)$ were determined using "Prism" software (GraphPad Software, San Diego, CA) for nonlinear regression to fit dose-response data to logistic curve models.

RNase $\mathbf{H}$ assay-RNase $\mathrm{H}$ activity was measured essentially as previously described. ${ }^{55}$ Full-length HIV RT was incubated with the RNA/DNA duplex substrateHTS-1 (RNA 5'gaucugagccugggagcu -3'-fluorescein annealed to DNA 3'-CTAGACTCGGACCCTCGA -5'Dabcyl), a high sensitivity duplex that assesses non-specific internal cleavage of the RNA strand.

HIV-1 cytoprotection assay ${ }^{35}$ - The HIV Cytoprotection assay used CEM-SS cells and the IIIB strain of HIV-1. Briefly virus and cells were mixed in the presence of test compound and incubated for 6 days. The virus was pre-titered such that control wells exhibit 70 to $95 \%$ loss of cell viability due to virus replication. Therefore, antiviral effect or cytoprotection was observed when compounds prevent virus replication. Each assay plate contained cell control wells (cells only), virus control wells (cells plus virus), compound toxicity control wells (cells plus compound only), compound colorimetric control wells (compound only) as well as experimental wells (compound plus cells plus virus). Cytoprotection and compound cytotoxicity were assessed by MTS (CellTiter® 96 Reagent, Promega, Madison WI) and the $\mathrm{EC}_{50}$ (concentration inhibiting virus replication by 50\%), $\mathrm{CC}_{50}$ (concentration resulting in 50\% cell death) and a calculated TI (therapeutic index $\mathrm{CC}_{50} / \mathrm{EC}_{50}$ ) were provided. Each assay included AZT as a positive control. 
Antiviral MAGI assays-MAGI assays were carried out using P4R5 indicator cells essentially as previously described. ${ }^{36}$ P4R5 cells were cultured in 96-well microplates with $4 \times 10^{3}$ cells per well and maintained in DMEM/10\% FBS supplemented with puromycin (1 $\mu \mathrm{g} / \mathrm{ml})$. Cells were incubated with either $1 \%$ DMSO or varying concentrations of the drugs for $24 \mathrm{~h}$ and then exposed to HIV (MOI of 1.25) followed by an additional incubation period of $48 \mathrm{~h}$. The extent of infection was assessed using a fluorescence-based $\beta$-galactosidase detection assay, as previously described with minor modifications. ${ }^{56}$ After the $48 \mathrm{~h}$ incubation period, cells were lysed and substrate 4-methylumbelliferyl-galactoside (MUG) was added. The $\beta$-galactosidase produced during infection acts on the substrate MUG and yields a fluorescent product 4-methylumbelliferone (4-MU) that could be detected fluorimetrically with excitation wavelength $365 \mathrm{~nm}$ and emission wavelength $446 \mathrm{~nm}$.

Modeling and docking-Molecular modeling was performed using the Schrodinger small molecule drug discovery suite 2014-3. The crystal structure of PFV intasome in complex with magnesium and $\mathbf{3}$ was used as a starting point (PDB code: $3 \mathrm{~S} 3 \mathrm{M}$ ). ${ }^{50}$ This model was subjected to Protein Preparation Wizard ${ }^{57-58}$ (Schrödinger Inc.) in which missing hydrogens atoms were added, zero-order bonds to metals were created followed by the generation of metal binding states. The structure of protein was minimized using OPLS 2005 force field ${ }^{59}$ to optimize hydrogen bonding network and converge heavy atoms to an rmsd of $0.3 \AA$. The receptor grid generation tool in Maestro (Schrodinger Inc.) was used to define an active site around the native ligand (3) to cover all the residues within $12 \AA$ from it with both the metal cofactors $\left(\mathrm{Mg}^{2+}\right)$ as a constraint to identify the chelating triad during docking.

All the compounds synthesized were drawn using Maestro and subjected to Lig Prep ${ }^{58}$ to generate conformers, possible protonation at $\mathrm{pH}$ of $7 \pm 3$, and metal binding states that serve as an input for docking process. All the dockings were performed using Glide $\mathrm{XP}^{60}$ (Glide, version 6.4) mode with both the $\mathrm{Mg}^{2+}$ metal cofactors as a constraint. The van der Waals radii of nonpolar atoms for each of the ligands were scaled by a factor of 0.8 .

To understand the effect of observed mutations on resistance profile for $\mathbf{1}, \mathbf{3}$ and the representative compound 45, a crystal structure of PFV intasome in complex with magnesium and $\mathbf{3}$ was used as a starting point (PDB code: 3S3M). The observed mutants were changed in the crystal structure manually using Maestro (PFV intasome numbering of G209S, Y212C, Q217H and N224H corresponds to HIV intasome numbering of G140S, Y143C, Q148H and N155H) followed by a protein preparation.

Three compounds $(\mathbf{1}, \mathbf{3}$ and $\mathbf{4 5})$ were drawn using Maestro and subjected to Lig Prep to generate conformers, possible protonation at $\mathrm{pH}$ of $7 \pm 3$, and metal binding states that serve as an input for docking process. These compounds were docked using a similar protocol as above.

\section{Supplementary Material}

Refer to Web version on PubMed Central for supplementary material. 


\section{ACKNOWLEDGMENTS}

This research was supported by the National Institutes of Health (AI100890) and partially by the Center for Drug Design, University of Minnesota.

\section{ABBREVIATIONS USED}

\begin{tabular}{|c|c|}
\hline HIV & human immunodeficiency virus \\
\hline INST & integrase strand transfer \\
\hline HPD & 3-hydroxypyrimidine-2,4-dione \\
\hline INST & integrase strand transfer \\
\hline INSTIs & integrase strand transfer inhibitors \\
\hline RT & reverse transcriptase \\
\hline HAART & highly active antiretroviral therapy \\
\hline NRTIs & nucleoside reverse transcriptase inhibitors \\
\hline NNRTIs & nonnucleoside reverse transcriptase inhibitors \\
\hline PIs & protease inhibitors \\
\hline DKA & diketoacid \\
\hline CPE & cytopathic effect \\
\hline SAR & structure-activity-relationship \\
\hline MOA & mechanism of action \\
\hline NTD & $\mathrm{N}$-terminal domain \\
\hline CCD & catalytic core domain \\
\hline CTD & C-terminal domain \\
\hline PFV & prototype foamy virus. \\
\hline
\end{tabular}

\section{References}

1. Kinch MS, Patridge E. An analysis of FDA-approved drugs for infectious disease: HIV/AIDS drugs. Drug Discov. Today. 2014; 19:1510-1513. [PubMed: 24880109]

2. Barouch DH, Deeks SG. Immunologic strategies for HIV-1 remission and eradication. Science. 2014; 345:169-174. [PubMed: 25013067]

3. Chun TW, Fauci AS. HIV reservoirs: pathogenesis and obstacles to viral eradication and cure. AIDS. 2012; 26:1261-1268. [PubMed: 22472858]

4. Bagasra O. A unified concept of HIV latency. Expert Opin. Biol. Ther. 2006; 6:1135-1149. [PubMed: 17049012]

5. Chun T-W, Stuyver L, Mizell SB, Ehler LA, Mican JAM, Baseler M, Lloyd AL, Nowak MA, Fauci AS. Presence of an inducible HIV-1 latent reservoir during highly active antiretroviral therapy. Proc. Natl. Acad. Sci. USA. 1997; 94:13193-13197. [PubMed: 9371822] 
6. Finzi D, Hermankova M, Pierson T, Carruth LM, Buck C, Chaisson RE, Quinn TC, Chadwick K, Margolick J, Brookmeyer R, Gallant J, Markowitz M, Ho DD, Richman DD, Siliciano RF. Identification of a reservoir for HIV-1 in patients on highly active antiretroviral therapy. Science. 1997; 278:1295-1300. [PubMed: 9360927]

7. Pommier Y, Johnson AA, Marchand C. Integrase inhibitors to treat HIV/AIDS. Nat. Rev. Drug Discovery. 2005; 4:236-248. [PubMed: 15729361]

8. Cotelle P. Patented HIV-1 integrase inhibitors (1998-2005). Recent Pat. Anti-Infect. Drug Discovery. 2006; 1:1-15.

9. Henao-Mejia J, Goez Y, Patino P, Rugeles MT. Diketo acids derivatives as integrase inhibitors: the war against the acquired immunodeficiency syndrome. Recent Pat.Anti-Infect. Drug Discovery. 2006; 1:255-265.

10. Dubey S, Satyanarayana YD, Lavania H. Development of integrase inhibitors for treatment of AIDS: An overview. Eur. J. Med. Chem. 2007; 42:1159-1168. [PubMed: 17367896]

11. Marchand C, Maddali K, Metifiot M, Pommier Y. HIV-1 IN inhibitors: 2010 update and perspectives. Curr. Top. Med. Chem. 2009; 9:1016-1037. [PubMed: 19747122]

12. Dayam R, Sanchez T, Neamati N. Diketo acid pharmacophore. 2. discovery of structurally diverse inhibitors of HIV-1 integrase. J. Med. Chem. 2005; 48:8009-8015. [PubMed: 16335925]

13. Dayam R, Sanchez T, Clement O, Shoemaker R, Sei S, Neamati N. b-Diketo acid pharmacophore hypothesis. 1. discovery of a novel class of HIV-1 integrase inhibitors. J. Med. Chem. 2005; 48:111-120. [PubMed: 15634005]

14. Deng J, Sanchez T, Neamati N, Briggs JM. Dynamic pharmacophore model optimization: identification of novel HIV-1 integrase inhibitors. J. Med. Chem. 2006; 49:1684-1692. [PubMed: 16509584]

15. Barreca ML, Ferro S, Rao A, De Luca L, Zappala M, Monforte A-M, Debyser Z, Witvrouw M, Chimirri A. Pharmacophore-based design of HIV-1 integrase strand-transfer inhibitors. J. Med. Chem. 2005; 48:7084-7088. [PubMed: 16250669]

16. Mustata GI, Brigo A, Briggs JM. HIV-1 integrase pharmacophore model derived from diverse classes of inhibitors. Bioorg. Med. Chem. Lett. 2004; 14:1447-1454. [PubMed: 15006380]

17. Carlson HA, Masukawa KM, Rubins K, Bushman FD, Jorgensen WL, Lins RD, Briggs JM, McCammon JA. Developing a dynamic pharmacophore model for HIV-1 integrase. J. Med. Chem. 2000; 43:2100-2114. [PubMed: 10841789]

18. Cocohoba J, Dong BJ. Raltegravir: the first HIV integrase inhibitor. Clin. Ther. 2008; 30:17471765. [PubMed: 19014832]

19. Summa V, Petrocchi A, Bonelli F, Crescenzi B, Donghi M, Ferrara M, Fiore F, Gardelli C, Gonzalez Paz O, Hazuda DJ, Jones P, Kinzel O, Laufer R, Monteagudo E, Muraglia E, Nizi E, Orvieto F, Pace P, Pescatore G, Scarpelli R, Stillmock K, Witmer MV, Rowley M. Discovery of raltegravir, a potent, selective orally bioavailable HIV-Integrase inhibitor for the treatment of HIVAIDS infection. J. Med. Chem. 2008; 51:5843-5855. [PubMed: 18763751]

20. Shimura K, Kodama EN. Elvitegravir: a new HIV integrase inhibitor. Antiviral Chem. Chemother. 2009; 20:79-85.

21. Vandekerckhove L. GSK-1349572, a novel integrase inhibitor for the treatment of HIV infection. Curr. Opin. Invest. Drugs. 2010; 11:203-212.

22. Min S, Song I, Borland J, Chen S, Lou Y, Fujiwara T, Piscitelli SC. Pharmacokinetics and safety of S/GSK1349572, a next-generation HIV integrase inhibitor, in healthy volunteers. Antimicrob. Agents Chemother. 2009; 54:254-258. [PubMed: 19884365]

23. Shafer RW. Rationale and uses of a public HIV drug-resistance database. J. Infect. Dis. 2006; 194(Suppl 1):S51-8. [PubMed: 16921473]

24. Tang J, Maddali K, Dreis CD, Sham YY, Vince R, Pommier Y, Wang Z. N-3 hydroxylation of pyrimidine-2,4-diones yields dual inhibitors of HIV reverse transcriptase and integrase. ACS Med. Chem. Lett. 2011; 2:63-67. [PubMed: 21499541]

25. Tang J, Maddali K, Dreis CD, Sham YY, Vince R, Pommier Y, Wang ZQ. 6-Benzoyl-3hydroxypyrimidine-2,4-diones as dual inhibitors of HIV reverse transcriptase and integrase. Bioorg. Med. Chem. Lett. 2011; 21:2400-2402. [PubMed: 21392991] 
26. Tang J, Maddali K, Metifiot M, Sham YY, Vince R, Pommier Y, Wang ZQ. 3Hydroxypyrimidine-2,4-diones as an inhibitor scaffold of HIV integrase. J. Med. Chem. 2011; 54:2282-2292. [PubMed: 21381765]

27. Tang J, Maddali K, Dreis CD, Sham YY, Vince R, Pommier Y, Wang ZQ. 6-Benzoyl-3hydroxypyrimidine-2,4-diones as dual inhibitors of HIV reverse transcriptase and integrase. Bioorg. Med. Chem. Lett. 2011; 21:2400-2402. [PubMed: 21392991]

28. Vernekar SK, Liu Z, Nagy E, Miller L, Kirby KA, Wilson DJ, Kankanala J, Sarafianos SG, Parniak MA, Wang Z. Design, synthesis, biochemical, and antiviral evaluations of C6 benzyl and C6 biarylmethyl substituted 2-hydroxylisoquinoline-1,3-diones: dual inhibition against HIV reverse transcriptase-associated RNase $\mathrm{H}$ and polymerase with antiviral activities. J. Med. Chem. 2015; 58:651-664. [PubMed: 25522204]

29. Mai X, Lu XS, Xia HY, Cao YS, Liao YJ, Lv XL. Synthesis, antitumor evaluation and crystal structure of hydroxyurea derivatives. Chem. Pharm. Bull. 2010; 58:94-97. [PubMed: 20045973]

30. Stadlbauer W, Badawey ES, Hojas G, Roschger P, Kappe T. Malonates in cyclocondensation reactions. Molecules. 2001; 6:338-352.

31. Arnott EA, Chan LC, Cox BG, Meyrick B, Phillips A. POCl3 chlorination of 4-quinazolones. J. Org. Chem. 2011; 76:1653-1661. [PubMed: 21294532]

32. Kotera M, Ishii K, Tamura O, Sakamoto M. 1,3-dipolar cycloadditions of photoinduced carbonyl ylides. Part 2. Photoreactions of alpha,beta-unsaturated gamma,delta-epoxy dinitriles and ethyl vinyl ether. J. Chem. Soc. Perk T. 1. 1998:313-318.

33. Lee IY, Lee JY, Gong YD. Microwave-assisted facile one-step carbamoylation of 6-aminouracils. Synthesis. 2005:2713-2717.

34. Fletcher S, Gunning PT. Mild, efficient and rapid O-debenzylation of ortho-substituted phenols with trifluoroacetic acid. Tetrahedron Lett. 2008; 49:4817-4819.

35. Mosmann T. Rapid colorimetric assay for cellular growth and survival: application to proliferation and cytotoxicity assays. J. Immunol. Methods. 1983; 65:55-63. [PubMed: 6606682]

36. Sirivolu VR, Vernekar SKV, Ilina T, Myshakina NS, Parniak MA, Wang ZQ. Clicking 3 'azidothymidine into novel potent inhibitors of human immunodeficiency virus. J. Med. Chem. 2013; 56:8765-8780. [PubMed: 24102161]

37. Kimpton J, Emerman M. Detection of replication-competent and pseudotyped human immunodeficiency virus with a sensitive cell line on the basis of activation of an integrated betagalactosidase gene. J. Virol. 1992; 66:2232-2239. [PubMed: 1548759]

38. Metifiot M, Marchand C, Maddali K, Pommier Y. Resistance to integrase inhibitors. Viruses. 2010; 2:1347-1366. [PubMed: 20706558]

39. Metifiot M, Maddali K, Naumova A, Zhang X, Marchand C, Pommier Y. Biochemical and pharmacological analyses of HIV-1 integrase flexible loop mutants resistant to raltegravir. Biochemistry. 2010; 49:3715-3722. [PubMed: 20334344]

40. da Silva D, Van Wesenbeeck L, Breilh D, Reigadas S, Anies G, Van Baelen K, Morlat P, Neau D, Dupon M, Wittkop L, Fleury H, Masquelier B. HIV-1 resistance patterns to integrase inhibitors in antiretroviral-experienced patients with virological failure on raltegravir-containing regimens. J. Antimicrob. Chemother. 2010; 65:1262-1269. [PubMed: 20388636]

41. Reuman EC, Bachmann MH, Varghese V, Fessel WJ, Shafer RW. Panel of prototypical raltegravirresistant infectious molecular clones in a novel integrase-deleted cloning vector. Antimicrob. Agents Chemother. 2010; 54:934-936. [PubMed: 19917747]

42. Fantauzzi A, Mezzaroma I. Dolutegravir: clinical efficacy and role in HIV therapy. Ther. Adv. Chronic Dis. 2014; 5:164-177. [PubMed: 24982751]

43. Nowotny M. Retroviral integrase superfamily: the structural perspective. EMBO Rep. 2009; 10:144-151. [PubMed: 19165139]

44. Esposito D, Craigie R. HIV integrase structure and function. Adv. Virus Res. 1999; 52:319-333. [PubMed: 10384240]

45. Goldgur Y, Dyda F, Hickman AB, Jenkins TM, Craigie R, Davies DR. Three new structures of the core domain of HIV-1 integrase: an active site that binds magnesium. Proc. Natl. Acad. Sci. USA. 1998; 95:9150-9154. [PubMed: 9689049] 
46. Dyda F, Hickman AB, Jenkins TM, Engelman A, Craigie R, Davies DR. Crystal structure of the catalytic domain of HIV-1 integrase: similarity to other polynucleotidyl transferases. Science. 1994; 266:1981-1986. [PubMed: 7801124]

47. O'Brien C. HIV integrase structure catalyzes drug search. Science. 1994; 266:1946. [PubMed: 7801119]

48. Hare S, Gupta SS, Valkov E, Engelman A, Cherepanov P. Retroviral intasome assembly and inhibition of DNA strand transfer. Nature. 2010; 464:232-236. [PubMed: 20118915]

49. Hare S, Vosb AM, Claytonb RF, Thuringb JW, Cummingsb MD, Cherepanov P. Molecular mechanisms of retroviral integrase inhibition and the evolution of viral resistance. Proc. Natl. Acad. Sci. USA. 2010; 157:20057-20062. [PubMed: 21030679]

50. Hare S, Smith SJ, Metifiot M, Jaxa-Chamiec A, Pommier Y, Hughes SH, Cherepanov P. Structural and functional analyses of the second-generation integrase strand transfer inhibitor dolutegravir (S/ GSK1349572). Mol. Pharmacol. 2011; 80:565-572. [PubMed: 21719464]

51. Krishnan L, Li X, Naraharisetty HL, Hare S, Cherepanov P, Engelman A. Structure-based modeling of the functional HIV-1 intasome and its inhibition. Proc. Natl. Acad. Sci. U. S. A. 2010; 107:15910-15915. [PubMed: 20733078]

52. Wang Z, Bennett EM, Wilson DJ, Salomon C, Vince R. Rationally designed dual inhibitors of HIV reverse transcriptase and integrase. J. Med. Chem. 2007; 50:3416-3419. [PubMed: 17608468]

53. Leh H, Brodin P, Bischerour J, Deprez E, Tauc P, Brochon JC, LeCam E, Coulaud D, Auclair C, Mouscadet JF. Determinants of Mg2+-dependent activities of recombinant human immunodeficiency virus type 1 integrase. Biochemistry. 2000; 39:9285-9294. [PubMed: 10924121]

54. Semenova EA, Johnson AA, Marchand C, Davis DA, Yarchoan R, Pommier Y. Preferential inhibition of the magnesium-dependent strand transfer reaction of HIV-1 integrase by ahydroxytropolones. Mol. Pharmacol. 2006; 69:1454-1460. [PubMed: 16418335]

55. Parniak MA, Min KL, Budihas SR, Le Grice SF, Beutler JA. A fluorescence-based highthroughput screening assay for inhibitors of human immunodeficiency virus-1 reverse transcriptase-associated ribonuclease H activity. Anal. Biochem. 2003; 322:33-39. [PubMed: 14705777]

56. Abram ME, Parniak MA. Virion instability of human immunodeficiency virus type 1 reverse transcriptase (RT) mutated in the protease cleavage site between RT p51 and the RT RNase H domain. J. Virol. 2005; 79:11952-11961. [PubMed: 16140771]

57. Sastry GM, Adzhigirey M, Day T, Annabhimoju R, Sherman W. Protein and ligand preparation: parameters, protocols, and influence on virtual screening enrichments. J. Comput. Aided Mol. Des. 2013; 27:221-234. [PubMed: 23579614]

58. Schrödinger Release 2014-3: Schrödinger Suite 2014-3 Protein Preparation Wizard; Epik version 2.9, Schrödinger. LLC; New York, NY: 2014. Impact version 6.4, Schrödinger, LLC, New York, NY, 2014; Prime version 3.7, Schrödinger, LLC, New York, NY, 2014. In

59. Jorgensen WL, Maxwell DS, TiradoRives J. Development and testing of the OPLS all-atom force field on conformational energetics and properties of organic liquids. J. Am. Chem. Soc. 1996; 118:11225-11236.

60. Friesner RA, Banks JL, Murphy RB, Halgren TA, Klicic JJ, Mainz DT, Repasky MP, Knoll EH, Shelley M, Perry JK, Shaw DE, Francis P, Shenkin PS. Glide: a new approach for rapid, accurate docking and scoring. 1. Method and assessment of docking accuracy. J. Med. Chem. 2004; 47:1139-1149. 


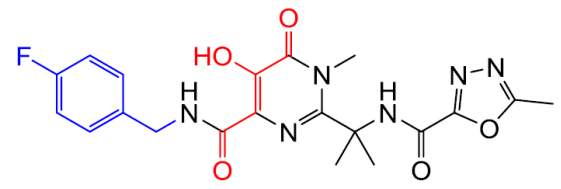

1

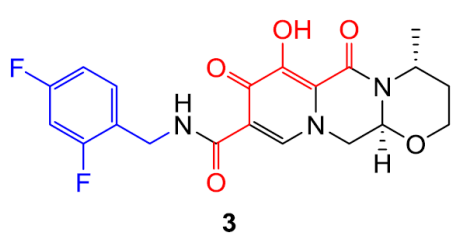

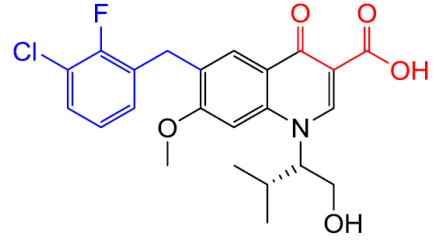

2

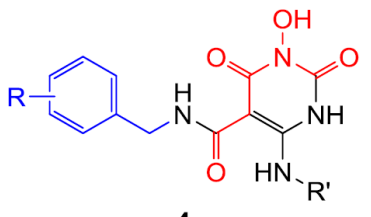

4

Figure 1.

Structures of FDA-approved INSTIs: raltegravir (1), elvitegravir (2), dolutegravir (3), and our newly designed HPD inhibitor subtype 4. Each approved drug features a chelating traid (red) and a terminal benzyl group (blue) that constitute the pharmacophore of HIV-1 INSTIs. Chemotype 4 fits the pharmacophore with the same two structural features. 

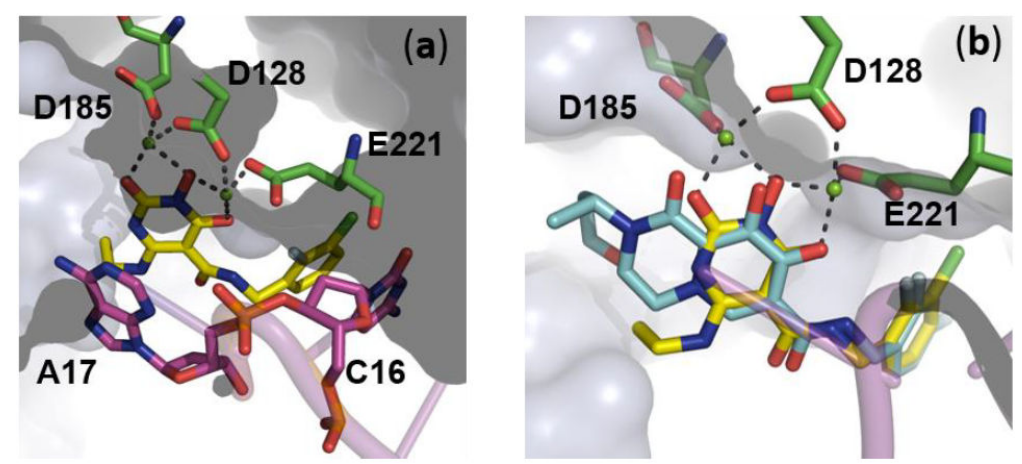

Figure 2.

a) Predicted binding mode of $\mathbf{4 5}$ (yellow) as obtained by molecular docking in the PFV intasome catalytic site (PDB code: $3 \mathrm{~S} 3 \mathrm{M}$ ). ${ }^{50}$ Magnesium cations depicted in green spheres, catalytic residues in green sticks and viral DNA in magenta cartoon. b) Overlay of 45 (yellow) and crystallographic $\mathbf{3}$ (cyan) in the catalytic active site of PFV intasome (PDB code: $3 \mathrm{~S} 3 \mathrm{M}$ ) with $\mathrm{Mg}^{2+}$ ions in green spheres and viral DNA in magenta cartoon.

Numbering in black is in accordance to the PFV intasome crystal structure (PDB code: 3S3M). 

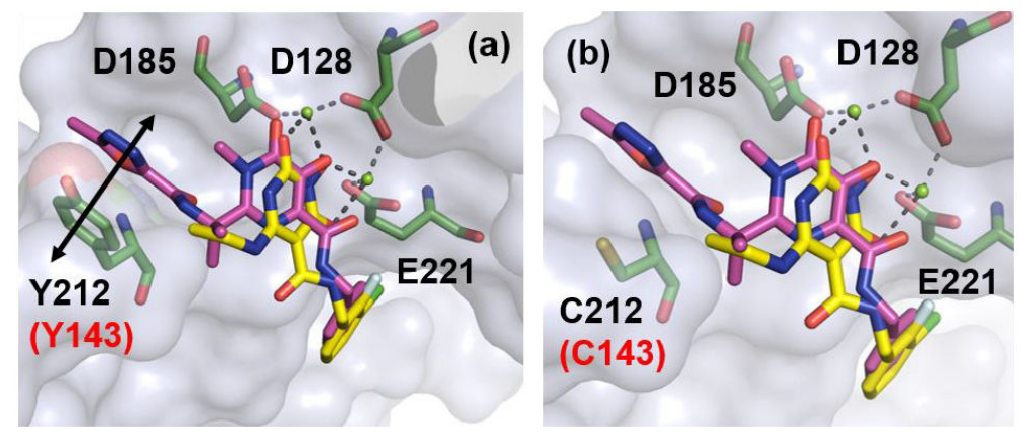

Figure 3.

a) Overlay of $\mathbf{1}$ (magenta) and $\mathbf{4 5}$ (yellow) within the PFV IN active site in complex with magnesium (green spheres) (PDB code: 3OYA). $\pi-\pi$ stack interaction between oxadiazole moiety within 1 and Y212 is shown by black double headed arrow. b) Overlay of $\mathbf{1}$ (magenta) and 45 (yellow) within the active site of PFV IN Y212C (corresponding to HIV IN Y143C) mutant. Key residues (D128, D185, E221 andY212) are represented as green sticks. Numbering in black is in accordance to the PFV intasome crystal structure (PDB code: $3 \mathrm{~S} 3 \mathrm{M}$ ). The corresponding numbering for HIV IN is highlighted in red. 


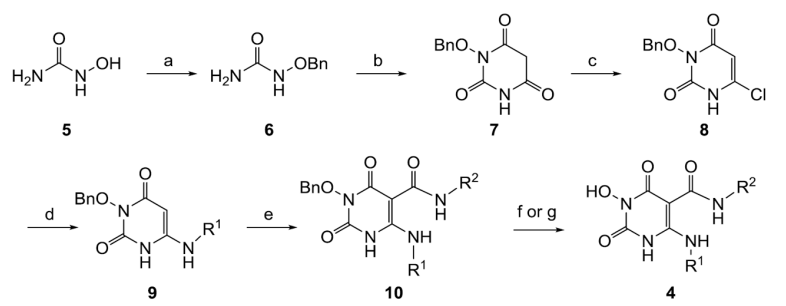

${ }^{a}$ Reagents and conditions: a) $\mathrm{KOH}, \mathrm{BnBr}, \mathrm{MeOH}$, reflux, 6 h, 91\%; b) $\mathrm{CH}_{2}(\mathrm{COOEt})_{2}$, $\mathrm{NaOEt}, \mathrm{MW}, 150^{\circ} \mathrm{C}, 20 \mathrm{~min}, 58 \%$; c) $\mathrm{POCl}_{3}, \mathrm{BnEt}_{3} \mathrm{NCl}, 50^{\circ} \mathrm{C}, 6 \mathrm{~h}, 88 \%$; d) $\mathrm{R}^{1}-\mathrm{NH}_{2}, \mathrm{~N}, \mathrm{~N}-$ dimethylaniline, $\mathrm{MW}, 170{ }^{\circ} \mathrm{C}, 20-25 \mathrm{~min}, 54-78 \%$; e) $\mathrm{R}^{2} \mathrm{NCO}$ (or $\mathrm{R}^{2} \mathrm{CON}_{3}$ ), $\mathrm{PhNO}_{2}$, $\mathrm{MW}$, $210^{\circ} \mathrm{C}, 20-40 \mathrm{~min}, 60-100 \%$; f) Pd/C, $\mathrm{H}_{2}, 40 \sim 50 \mathrm{Psi}, \mathrm{MeOH}, 4-6$ h, 82-97\%; g) TFA, MW, $120^{\circ} \mathrm{C}, 35-55 \mathrm{~min}, 50-77 \%$.

Scheme 1a.

Synthesis of chemotype 4 


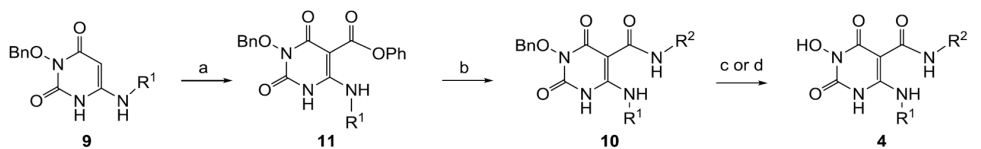

aReagents and conditions: a) $\mathrm{ClCO}_{2} \mathrm{Ph}$, pyridine, $\mathrm{rt}, 2 \mathrm{~h}, 62 \%$; b) $\mathrm{R}^{2}-\mathrm{NH}_{2}$, MW, dioxane, $100{ }^{\circ} \mathrm{C}, 50 \mathrm{~min}, 82-92 \%$; c) Pd/C, H2, 40 50 Psi, $\mathrm{MeOH}, 4-6$ h, 82-97\%; d) TFA, MW, $120^{\circ} \mathrm{C}, 35-55 \min , 50-77 \%$.

Scheme 2a.

Alternative synthesis of chemotype 4 
(a)

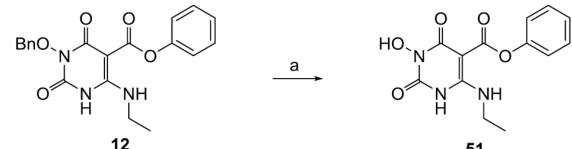

(b)
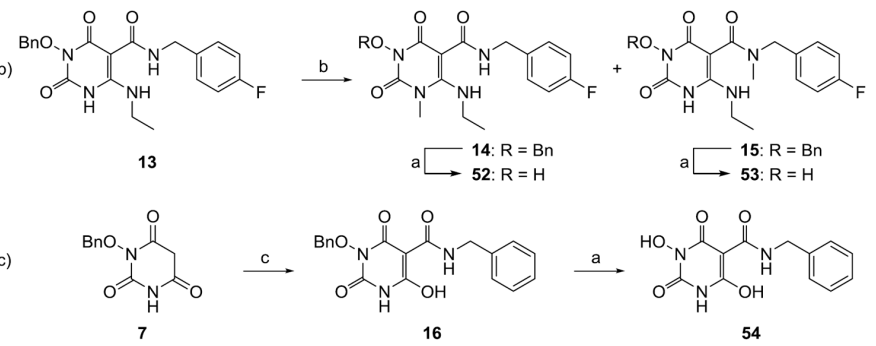

a Reagents and conditions: a) TFA, MW, $120{ }^{\circ} \mathrm{C}, 35-55 \mathrm{~min}$; b) $\mathrm{MeI}, \mathrm{Cs}_{2} \mathrm{CO}_{3}, \mathrm{DMSO}$, rt,

2h; c) benzyl isocyanate, ${ }^{i} \operatorname{Pr}_{2} \mathrm{NEt}, \mathrm{DCM}, \mathrm{rt}, 3 \mathrm{~h}$.

Scheme 3a.

Synthesis of compounds 51-54 


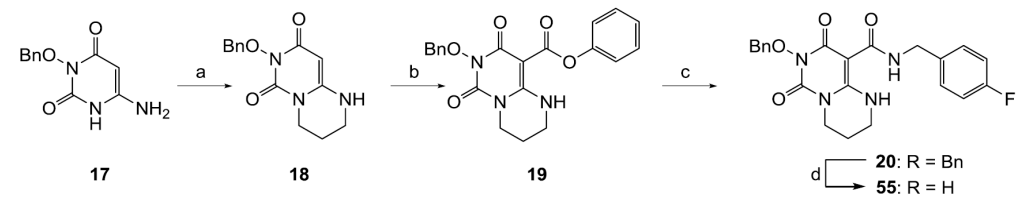

${ }^{a}$ Reagents and conditions: a) 1,3-dibromopropane, $\mathrm{K}_{2} \mathrm{CO}_{3}$, DMF, $60{ }^{\circ} \mathrm{C}, 2 \mathrm{~h}, 90 \%$; b) phenyl chloroformate, pyridine, rt, $3 \mathrm{~h}, 86 \%$; c) 4-fluorobenzylamine, $\mathrm{MW}$, dioxane, $100{ }^{\circ} \mathrm{C}, 50$ $\min , 91 \%$; d) TFA, MW, $120^{\circ} \mathrm{C}, 35 \mathrm{~min}, 96 \%$.

Scheme 4a.

Synthesis of compound $\mathbf{5 5}$ 


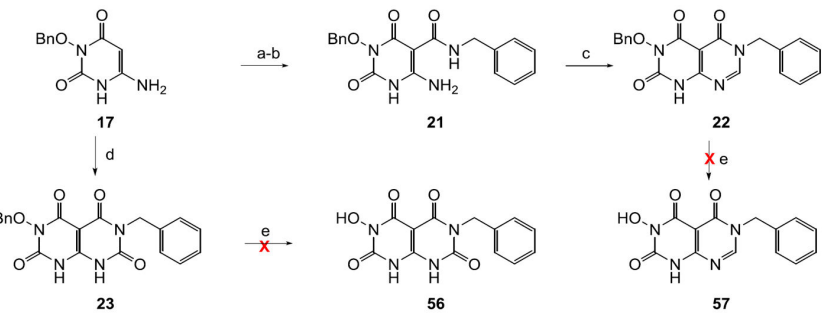

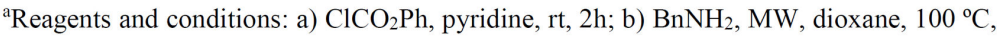
50 min, $76 \%$; c) $\mathrm{HC}(\mathrm{OEt})_{3}, \mathrm{CF}_{3} \mathrm{CO}_{2} \mathrm{H}$ (cat.), $\mathrm{MW} 150{ }^{\circ} \mathrm{C}, 10 \mathrm{~min}, 49 \%$; d) $\mathrm{BnNCO}$,

$\mathrm{PhNO}_{2}$, MW $210^{\circ} \mathrm{C}, 10 \mathrm{~min}, 62 \%$; e) TFA, MW, $120^{\circ} \mathrm{C}, 35-55 \mathrm{~min}$; or $\mathrm{Pd} / \mathrm{C}, \mathrm{H}_{2}, 40 \sim 50$

Psi, $\mathrm{MeOH}, 4-6 \mathrm{~h}$.

Scheme 5a.

Attempted synthesis of compounds 56-57 


\section{Table 1}

SAR of the C6 amino group: antiviral screening and biochemical assay of 24-36

\begin{tabular}{|c|c|c|c|c|}
\hline \multirow[t]{2}{*}{ Compd } & \multirow[t]{2}{*}{ Structure } & \multicolumn{2}{|c|}{ Antiviral Screening $(10 \mu \mathrm{M}){ }^{a}$} & \multirow{2}{*}{$\begin{array}{c}\text { INST IC }_{50} \\
(\mu \mathrm{M})^{b}\end{array}$} \\
\hline & & CPE reduction \% & viability $\%$ & \\
\hline 24 & & 0 & 21 & $>100$ \\
\hline 25 & & 0 & 13 & $>100$ \\
\hline 26 & & 100 & 98 & $0.080 \pm 0.007$ \\
\hline 27 & & 100 & 95 & $0.25 \pm 0.048$ \\
\hline 28 & & 100 & 89 & $0.11 \pm 0.021$ \\
\hline 29 & & 100 & 100 & $0.11 \pm 0.033$ \\
\hline 30 & & 100 & 100 & $0.033 \pm 0.004$ \\
\hline 31 & & 100 & 97 & $0.066 \pm 0.016$ \\
\hline 32 & & 33 & 48 & $0.058 \pm 0.012$ \\
\hline
\end{tabular}




\begin{tabular}{|c|c|c|c|c|}
\hline \multirow[t]{2}{*}{ Compd } & \multirow[t]{2}{*}{ Structure } & \multicolumn{2}{|c|}{ Antiviral Screening $(10 \mu \mathrm{M})^{a}$} & \multirow{2}{*}{$\begin{array}{c}\text { INST IC }_{50} \\
(\mu \mathrm{M})^{b}\end{array}$} \\
\hline & & CPE reduction \% & viability $\%$ & \\
\hline 33 & & 98 & 90 & $0.15 \pm 0.034$ \\
\hline 34 & & 98 & 91 & $0.21 \pm 0.077$ \\
\hline 35 & & 0 & 17 & $0.081 \pm 0.015$ \\
\hline 36 & & 99 & 100 & $0.90 \pm 0.29$ \\
\hline 3 & -- & -- & -- & $0.068 \pm 0.01$ \\
\hline 1 & -- & -- & -- & $0.65 \pm 0.14$ \\
\hline
\end{tabular}

${ }^{a}$ Cytoprotection assay using HIV-1 IIIB in CEM-SS cells with a viral control for CPE reduction and cell control for cell viability.

${ }^{b}$ Concentration inhibiting INST by $50 \%$, expressed as mean \pm standard deviation from at least three independent experiments. 


\section{Table 2}

SAR around the C5 carboxamide: antiviral screening and biochemical assay of $\mathbf{3 0}$ analogues $\mathbf{4 7 - 5 1}$

\begin{tabular}{|c|c|c|c|c|}
\hline \multirow[t]{2}{*}{ Compd } & \multirow[t]{2}{*}{ Structure } & \multicolumn{2}{|c|}{ Antiviral Screening $(10 \mu \mathrm{M}){ }^{a}$} & \multirow{2}{*}{$\begin{array}{l}\text { INST IC }_{50} \\
\qquad(\mu \mathrm{M})^{b}\end{array}$} \\
\hline & & CPE reduction \% & viability \% & \\
\hline 30 & & 100 & 100 & $0.033 \pm 0.004$ \\
\hline 37 & & 100 & 90 & $0.068 \pm 0.007$ \\
\hline 38 & & 100 & 99 & $0.049 \pm 0.004$ \\
\hline 39 & & 92 & 87 & $0.068 \pm 0.011$ \\
\hline 40 & & 100 & 92 & $0.048 \pm 0.007$ \\
\hline 41 & & 100 & 100 & $0.23 \pm 0.062$ \\
\hline 42 & & 100 & 100 & $0.077 \pm 0.011$ \\
\hline 43 & & 62 & 73 & $0.095 \pm 0.019$ \\
\hline 44 & & 100 & 100 & $0.12 \pm 0.021$ \\
\hline 45 & & 100 & 100 & $0.021 \pm 0.002$ \\
\hline
\end{tabular}




\begin{tabular}{|c|c|c|c|c|}
\hline \multirow[t]{2}{*}{ Compd } & \multirow[t]{2}{*}{ Structure } & \multicolumn{2}{|c|}{ Antiviral Screening $(10 \mu \mathrm{M})^{a}$} & \multirow{2}{*}{$\begin{array}{c}\text { INST IC }_{50} \\
\quad(\mu \mathrm{M})^{b}\end{array}$} \\
\hline & & CPE reduction \% & viability $\%$ & \\
\hline 46 & & 100 & 100 & $0.051 \pm 0.013$ \\
\hline 47 & & 99 & 87 & $0.10 \pm 0.03$ \\
\hline 48 & & 11 & 27 & $35 \pm 17$ \\
\hline 49 & & -- & -- & $>100$ \\
\hline $\mathbf{5 0}$ & & 5.1 & 100 & $>100$ \\
\hline 51 & & 50 & 100 & $>100$ \\
\hline 3 & -- & -- & -- & $0.068 \pm 0.01$ \\
\hline 1 & -- & -- & -- & $0.65 \pm 0.14$ \\
\hline
\end{tabular}

${ }^{a}$ Cytoprotection assay using HIV-1 IIIB in CEM-SS cells with a viral control for CPE reduction and cell control for cell viability. ${ }^{b}$ Concentration inhibiting INST by $50 \%$, expressed as mean \pm standard deviation from at least three independent experiments. 
Table 3

Antiviral screening and biochemical assay of scaffold analogues 52-55

\begin{tabular}{|c|c|c|c|c|}
\hline \multirow[t]{2}{*}{ Compd } & \multirow[t]{2}{*}{ Structure } & \multicolumn{2}{|c|}{ Antiviral Screening $(10 \mu \mathrm{M})^{a}$} & \multirow{2}{*}{$\begin{array}{c}\text { INST IC }_{50} \\
(\mu \mathrm{M})\end{array}$} \\
\hline & & CPE reduction \% & viability $\%$ & \\
\hline 52 & & 6.5 & 10 & $>100$ \\
\hline 53 & & 0 & 89 & $7.0 \pm 3.3$ \\
\hline 54 & & 26 & 100 & $37 \pm 21$ \\
\hline 55 & & 0 & 5.5 & $0.43 \pm 0.13$ \\
\hline
\end{tabular}


Table 4

Dose-response antiviral results of selected analogues from two different assays

\begin{tabular}{ccccc}
\hline \multirow{2}{*}{ Compound } & \multicolumn{3}{c}{ Cytoprotection Assay $^{\boldsymbol{a}}$} & \multirow{2}{*}{ MAGI Assay $^{\mathbf{b}}$} \\
\cline { 2 - 4 } & $\mathbf{E C}_{\mathbf{5 0}}(\boldsymbol{\mu M})^{\boldsymbol{c}}$ & $\mathbf{C C}_{\mathbf{5 0}}(\boldsymbol{\mu M})^{\boldsymbol{d}}$ & $\mathbf{T I}^{\boldsymbol{e}}$ & $\mathbf{E C}_{\mathbf{5 0}}(\boldsymbol{\mu M})^{\boldsymbol{f}}$ \\
\hline $\mathbf{2 6}$ & 0.83 & 58 & 70 & $3.7 \pm 0.3$ \\
$\mathbf{3 0}$ & 0.061 & 23 & 380 & $0.30 \pm 0.01$ \\
$\mathbf{3 8}$ & 0.033 & 19 & 580 & $0.090 \pm 0.02$ \\
$\mathbf{4 0}$ & 0.032 & 47 & 1500 & $0.070 \pm 0.02$ \\
$\mathbf{4 2}$ & 0.049 & 21 & 430 & $0.30 \pm 0.06$ \\
$\mathbf{4 4}$ & 0.19 & 19 & 100 & $0.80 \pm 0.2$ \\
$\mathbf{4 5}$ & 0.015 & 18 & 1200 & $0.30 \pm 0.09$ \\
$\mathbf{4 6}$ & 0.15 & 20 & 130 & $1.3 \pm 0.2$ \\
$\mathbf{3}$ & -- & -- & -- & $0.020 \pm 0.002$ \\
$\mathbf{1}$ & 0.023 & $>10$ & $>430$ & $0.030 \pm 0.008$ \\
\hline
\end{tabular}

${ }^{a}$ Concentration inhibiting INST by $50 \%$,

${ }^{c}$ Concentration of a compound inhibiting virus replication by $50 \%$.

${ }^{d}$ Concentration of a compound resulting in $50 \%$ cell death.

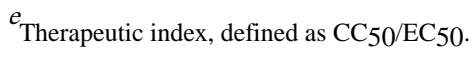

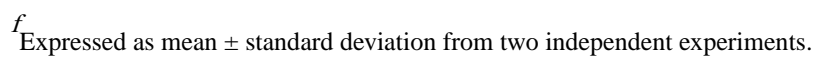


Table 5

Resistance profile of $\mathbf{4 5}$ against raltegravir-resistant HIV-1 clones

\begin{tabular}{cccccc}
\hline \multirow{2}{*}{ HIV-1 Clone } & Major Mutations & \multicolumn{4}{c}{ Fold-resistance } \\
\cline { 3 - 6 } & & $\mathbf{4 5}$ & AZT & $\mathbf{1}$ & $\mathbf{3}$ \\
\hline $1556-1$ & Y143C & 0.4 & 1.4 & 170 & 2.0 \\
$4736-2$ & N155H & 2.4 & 0.7 & 14 & 1.8 \\
$8070-1$ & G140S / Y143H / Q148H & 6.3 & 0.9 & 220 & 9.6 \\
$8070-2$ & G140S / Q148H & 13 & 0.5 & 145 & 12 \\
\hline
\end{tabular}

${ }^{a}$ Fold-resistance is defined as EC50(mutant) / EC50 (WT). 


\section{Table 6}

Biochemical inhibition of RT-associated RNase $\mathrm{H}$ and the antiviral correlation

\begin{tabular}{ccccc}
\hline \multirow{2}{*}{ Compound } & RNase $\mathbf{H}$ & INST & $\mathbf{C P E}$ & MAGI \\
& $\mathbf{I C}_{\mathbf{5 0}}(\boldsymbol{\mu M})^{\boldsymbol{a}}$ & $\mathbf{I C}_{\mathbf{5 0}}(\boldsymbol{\mu M})^{\boldsymbol{a}}$ & $\mathbf{E C}_{\mathbf{5 0}}(\boldsymbol{\mu M})^{\boldsymbol{b}}$ & $\mathbf{E C}_{\mathbf{5 0}}(\boldsymbol{\mu M})^{\boldsymbol{c}}$ \\
\hline $\mathbf{2 6}$ & $0.061 \pm 0.004$ & $0.080 \pm 0.007$ & 0.83 & $3.7 \pm 0.3$ \\
$\mathbf{3 0}$ & $0.042 \pm 0.012$ & $0.033 \pm 0.004$ & 0.061 & $0.30 \pm 0.01$ \\
$\mathbf{3 8}$ & $0.011 \pm 0.003$ & $0.049 \pm 0.004$ & 0.033 & $0.09 \pm 0.02$ \\
$\mathbf{4 0}$ & $0.010 \pm 0.006$ & $0.068 \pm 0.011$ & 0.032 & $0.07 \pm 0.02$ \\
$\mathbf{4 2}$ & $0.026 \pm 0.009$ & $0.077 \pm 0.011$ & 0.049 & $0.30 \pm 0.06$ \\
$\mathbf{4 4}$ & $0.022 \pm 0.009$ & $0.12 \pm 0.021$ & 0.19 & $0.80 \pm 0.2$ \\
$\mathbf{4 5}$ & $0.029 \pm 0.015$ & $0.021 \pm 0.002$ & 0.015 & $0.30 \pm 0.09$ \\
$\mathbf{4 6}$ & $0.020 \pm 0.010$ & $0.051 \pm 0.013$ & 0.15 & $1.3 \pm 0.2$ \\
$\mathbf{3}$ & $>10$ & $0.068 \pm 0.01$ & -- & $0.020 \pm 0.002$ \\
$\mathbf{1}$ & $>10$ & $0.65 \pm 0.14$ & 0.023 & $0.030 \pm 0.008$ \\
\hline
\end{tabular}

${ }^{a}$ Concentration with 50\% enzyme inhibition, expressed as mean \pm standard deviation from three independent experiments.

${ }^{b}$ Concentration of a compound inhibiting virus replication by $50 \%$.

${ }^{c}$ Concentration of a compound inhibiting virus replication by $50 \%$, expressed as mean \pm standard deviation from three independent experiments. 
Table 7

Physicochemical and in vitro ADME profile of selected analogues

\begin{tabular}{|c|c|c|c|c|c|c|c|c|}
\hline \multirow[t]{2}{*}{ Compd } & \multirow[t]{2}{*}{ Aqueous Solubility ( $\mu \mathrm{M})$} & \multirow[t]{2}{*}{$\begin{array}{c}\text { Aqueous } \\
\text { Stability } \\
\mathbf{t}_{\mathbf{1} / 2}(\mathbf{h})\end{array}$} & \multicolumn{2}{|c|}{$\begin{array}{c}\text { Plasma Stability } \mathbf{t}_{1 / 2} \\
\text { (h) }\end{array}$} & \multicolumn{2}{|c|}{$\begin{array}{l}\text { Plasma Protein } \\
\text { Binding }(\%)^{a}\end{array}$} & \multicolumn{2}{|c|}{$\begin{array}{c}\text { Microsomal Stability (Phase I / } \\
\left.\text { Phase II, } \mathbf{C L}_{\text {int }}\right)^{b}\end{array}$} \\
\hline & & & Human & Mouse & Human & Mouse & Human & Mouse \\
\hline 26 & 138 & $>24$ & $>24$ & $>24$ & 99.2 & 70.9 & $0.8 / 51$ & $<0.1 / 500$ \\
\hline 30 & 1091 & $>24$ & $>24$ & $>24$ & 99.1 & 83.9 & $<0.1 / 355$ & $<0.1 / 732$ \\
\hline 38 & 150 & $>24$ & $>24$ & $>24$ & 99.4 & 86.5 & $<0.1 / 336$ & $<0.1 / 1240$ \\
\hline 45 & 11 & $>24$ & $>24$ & $>24$ & 98.4 & 94.0 & $<0.1 / 850$ & $<0.1 / 1065$ \\
\hline 46 & 81 & $>24$ & $>24$ & $>24$ & 98.9 & 95.9 & $<0.1 / 357$ & $0.4 / 1342$ \\
\hline
\end{tabular}

${ }^{a}$ Percent of fraction bound.

${ }^{b} L_{\text {int }}$ intrinsic clearance, $\mu \mathrm{l} / \mathrm{min} / \mathrm{mg}$ protein. 